\title{
Total Sky Imager Model 880 Status and Testing Results
}

November 2001

C. N. Long ${ }^{(1)}$

D. W. Slater ${ }^{(1)}$

T. Tooman ${ }^{(2)}$

${ }^{(1)}$ Pacific Northwest National Laboratory, Richland, Washington

${ }^{(2)}$ Sandia National Laboratory, Livermore, California

Work supported by the U.S. Department of Energy,

Office of Energy Research, Office of Health and Environmental Research 


\section{Contents}

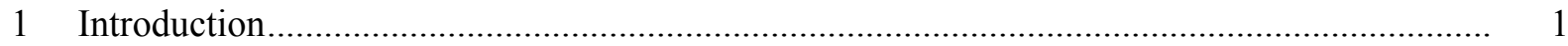

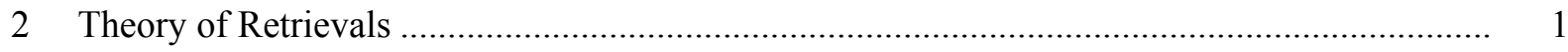

3 TSI Operation and Monitoring During Testing and Evaluation ............................................ 4

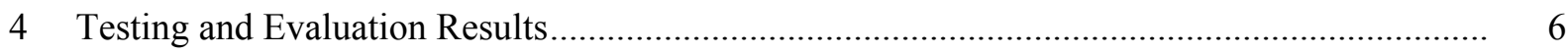

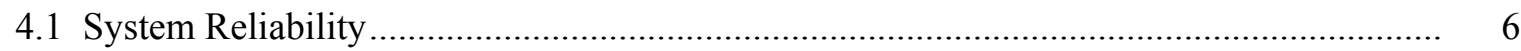

4.2 Comparison of Sky Cover Retrievals ...................................................................... 8

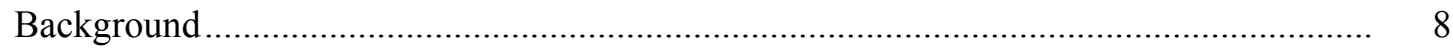

Procedure for TSI/WSI Sky Cover Comparisons …....................................................... 9

TSI/WSI Comparison Results.................................................................................... 10

Summary of Comparison Results ............................................................................ 12

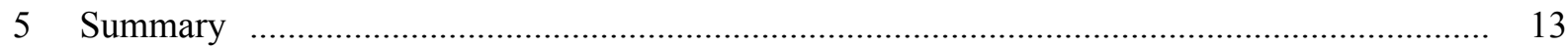

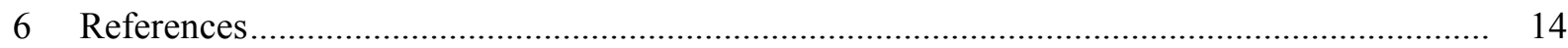

Appendix A - TSI 880 Operating Specifications .................................................................... A.1

Appendix B - TSI Operation and Monitoring Features …............................................... B. 1

Appendix C - Testing and Evaluation Data Formats …........................................................... C.1

\section{Figures}

1 The TSI 880 Pictured Here at the SGP CF.

2 Clear-Sky Image, Corresponding Relative Red/Blue Ratio "Image," and Separated Blue and Red Pixel Value Amount Images .................................................................................. 3

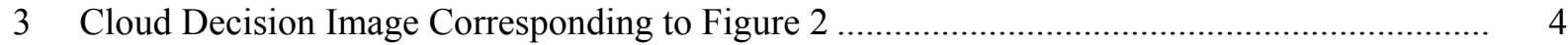

4 Cloudy-Sky Image, Corresponding Relative Red/Blue Ratio "Image," and Separated Blue and Red Pixel Value Amount Images ................................................................................... 5

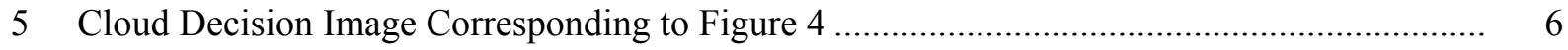

6 Picture Showing the Relative Closeness of the TSI and WSI at the SGP ............................... 7

7 Plot of the Actual and Possible Number of TSI Daylight Retrievals of Fractional Sky Cover at the SGP from July 1 through December 31, 2000 4 (

\section{7}


8 Plot of the Actual Divided by the Possible Number of TSI Daylight Retrievals of Fractional

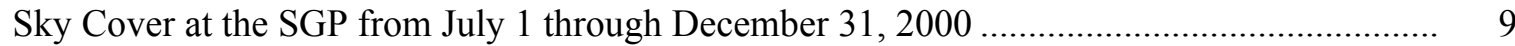

9 Plot of WSI Sky Cover Versus TSI Total and Opaque Sky Cover.......................................... 10

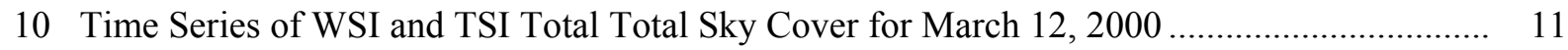

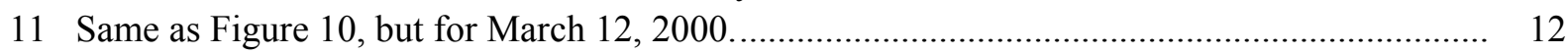

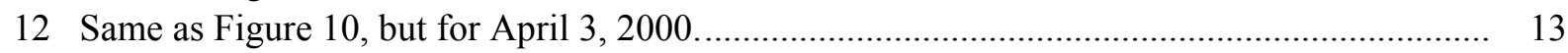

13 Plot of WSI - TSI Total Sky Cover Difference Versus TSI Total Sky Cover Amount............. 14

14 Plot of WSI - TSI Total Sky Cover Difference Versus TSI Thin Sky Cover Amount.............. 15

15 Plot of WSI - TSI Total Sky Cover Difference Versus Time of Day. ...................................... 16

16 Histogram of WSI - TSI Sky Cover Percent Difference for this Study in Terms of Number

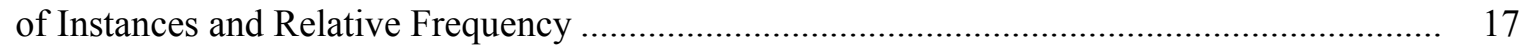




\section{Introduction}

The Total Sky Imager (TSI) is manufactured by Yankee Environmental Systems (YES) Incorporated, based in Turner Falls, Massachusetts. (For more information about YES, see http://www.yesinc.com/.) The TSI is a commercialized version of the Hemispheric Sky Imager prototype (Long et al. 1998). YES has now produced a more sophisticated (compared to the original model 440) model 880 of the TSI (see Figure 1). The first YES TSI 880 was deployed at the Blackwell Tonkawa Airport (BTA) as part of the Department of Energy (DOE) Atmospheric Radiation Measurement (ARM) Program 2000 Cloud intensive operational period (IOP). This TSI 880 collected data from March 2, 2000 through April 6, 2000. This report gives an assessment of the TSI based on the BTA and Southern Great Plane (SGP) Central Facility (CF) data collected to date.

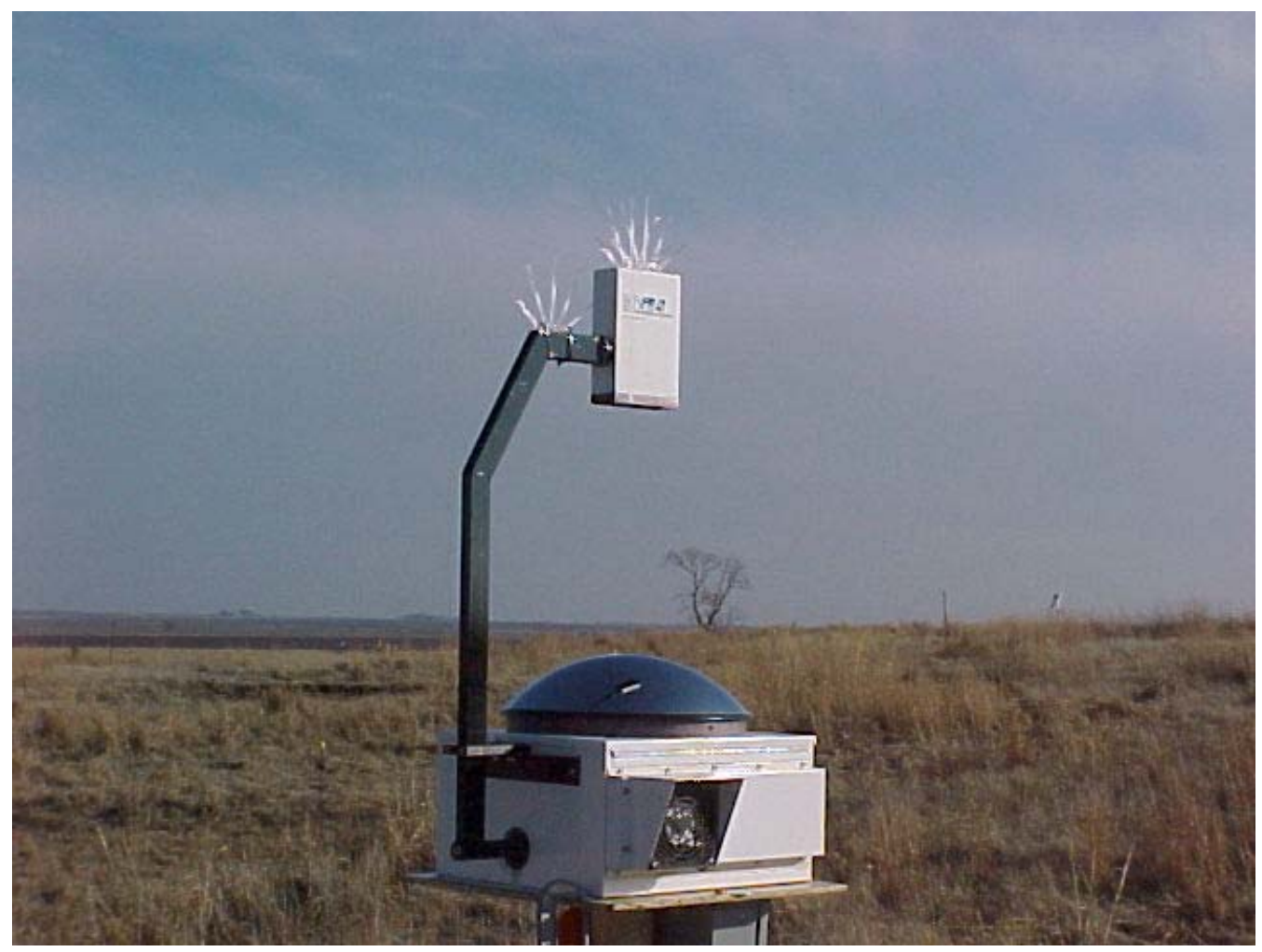

Figure 1. The TSI 880 Pictured Here at the SGP CF.

\section{Theory of Retrievals}

The TSI 880 captures images of the sky during daylight hours. These sky images are 24-bit color JPEG format at $352 \times 288$ pixel resolution. (More information on TSI 880 specs. is given in Appendix 1.) These "raw" sky images are processed to infer fractional sky cover. Note that the sky cover retrievals are generally only valid for solar elevation angles greater than $10^{\circ}$ (zenith angles less than $80^{\circ}$ ), and images are processed for a $160^{\circ}$ field-of-view (FOV), ignoring the $10^{\circ}$ of sky near the horizon. 
For molecular scattering (clear skies), more blue light is scattered than red, which is why the clearsky appears blue to our eyes. A sample image of clear-sky is shown in Figure 2. Below this sample sky image (Figure 2) are two images that show the corresponding extracted blue and red pixel values that make up the sample image. As is shown, the red pixel values are relatively small (dark) in the sky portion of the image compared to the corresponding blue pixel values, except near the horizon where the increased atmospheric path length makes the original sky image appear white to our eyes. The relative $\mathrm{red} / \mathrm{blue}$ ratio values are shown in the upper right image in Figure 2 and for clear-sky the red/blue ratio is small, i.e., dark in the image. Figure 3 shows the "cloud decision image" corresponding to Figure 2, a graphical depiction of the results of the sky cover retrieval processing. In the cloud decision image, the camera arm and sun-blocking strip are masked out with black, and the blue color in the rest of the circle denotes that all the processed pixels (for a $160^{\circ} \mathrm{FOV}$ ) have been determined to be "clear".

Clouds, unlike the clear sky, generally scatter both the blue and red visible light more equally. A sample of a partly cloudy-sky is shown in Figure 4. As in Figure 2, below this sample cloudy-sky image are two images that show the corresponding extracted blue and red pixel values that make up the sample image. In this case, where there are clouds present, the red pixel values are much greater than where there are not clouds. The blue pixel image shows far less contrast in pixel values. The relative ratio of red/blue pixel values (Figure 4 upper right) clearly shows that the ratio is greater for clouds than for clear-sky. We set a lower limit for clear-sky ratio value for each pixel in the image, and the pixels for which the red/blue ratio exceeds the clear limit are counted as "cloudy". The results of this processing are depicted in the "cloud decision image" shown in Figure 5, again with the camera arm and sun blocking strip masked out. In this image, the blue represents retrieved clear-sky, and the gray and white depict retrieved thin and opaque cloud, respectively. The estimated fractional sky cover is then calculated as the number of cloudy pixels divided by the total number of pixels in the $160^{\circ} \mathrm{FOV}$. Note that the TSI- 880 retrieval algorithm does not attempt to correct pixels for their view angle bias. That is, each pixel in the imager has a slightly different solid angle view of the sky as compared to adjacent pixels. The TSI- 880 retrieval does not attempt to bias or weight any pixels during processing for sky cover.

Included in the sky cover retrievals are separate accounting of the total number of pixels, and the detected thick (opaque) and thin cloud pixel counts, for a circle at zenith with respect to the sky imager (Figures 3 and 5). This separate "zenith circle" retrieval will facilitate comparison with vertically pointing instruments such as the Millimeter Wave Cloud Radar. In addition, separate cloud and clear pixel counts are retrieved for a circle around the position of the sun in the image (sun position is the small green cross on the sun strip mask in Figures 3 and 5), and for an angular area centered on the solar azimuth angle. These last two areas are problematic in sky images due to the limited intensity range of charged-coupled device (CCD) technology. Where our eyes can easily distinguish a circumsolar haze, which appears white but transparent to our eyes near the sun under hazy and/or sub-visual cirrus conditions, the sky in these parts of the sky image is often solid white. This is because the imager camera, as with many digital cameras, has a setting for "brightness" to make the images brighter or darker. The TSI brightness setting must be set high enough for the imager to "see" thin cloud. However, the limited intensity range of the camera CCD when used for sky imagery often results in the CCD elements representing the circumsolar area being overloaded, i.e., the intensity is beyond the maximum value the CCD element can handle. Thus, the circumsolar area of the image 


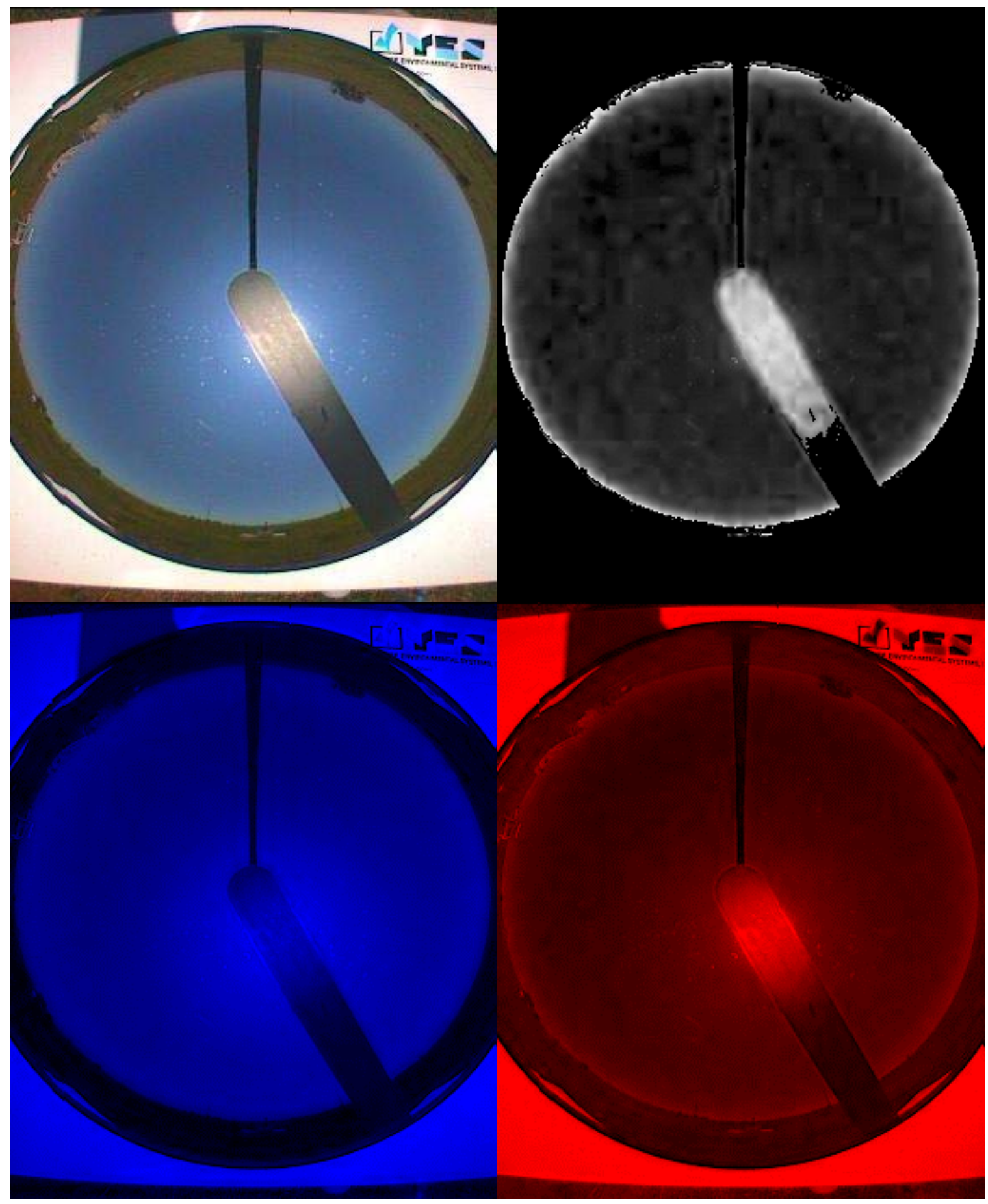

Figure 2. Clear-Sky Image (upper left), Corresponding Relative Red/Blue Ratio "Image" (upper right), Separated Blue (lower left), and Red (lower right) Pixel Value Amount Images. 


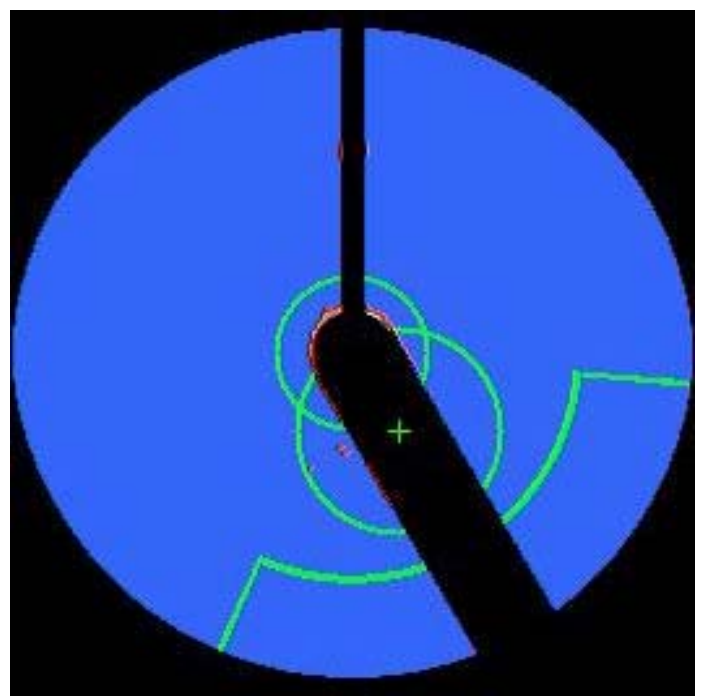

Figure 3. Cloud Decision Image Corresponding to Figure 2. Black denotes masking. Green outlines denote areas where separate cloud/clear pixel counts are included in the sky cover retrieval output files. Cloud decision color scheme is clear (blue), thin cloud (gray) and opaque cloud (white).

appears "white." A second stage of processing is currently under development that will attempt to determine through temporal statistics whether the "sun circle" and "horizon area" is being subjected to CCD overload, or whether there are actually clouds present in these areas of the image. Preliminary results have been encouraging, and it is expected that this second stage processing will improve the accuracy of the sky cover retrievals under these conditions.

\section{TSI Operation and Monitoring During Testing and Evaluation}

The TSI 880 is a daylight-only system, set to capture images from sunrise to sunset (solar zenith angle $\leq 90^{\circ}$ ). Sky cover retrievals, however, are only valid for solar elevation angles of $10^{\circ}$ or greater (solar zenith angle $\leq 80^{\circ}$ ). Within these ranges, sky images are normally captured and processed once per minute. During some parts of the evaluation period, image capture and processing were set at a rate of once every 30 seconds as a test. For the "normal" 1-minute capture rate, on board data storage includes about 2 days of "hot" storage (more on this in section 4a), and up to 2 weeks in the system removable Data Storage Module. (For more information about the Data Storage Module system, see http://www.yesinc.com/products/img.html.) The TSI 880 system includes a Web browser based user interface for both operating and monitoring the system performance. For the purposes of the testing and evaluation, the TSI 880 has been operated (processing limits, rate of capture, data ingest and storage, etc.) and monitored from PNNL (Pacific Northwest National Laboratory, Richland, Washington). (For more information on the TSI 880 Web based operation and monitoring capabilities, see Appendix 2.) Onsite operations include only daily cleaning of the mirror surface, and at one point installation of "bird spikes." This last was necessary due to the tendency of some local birds to perch on the top of the camera housing and horizontal portion of the camera arm. The result of this perching was a daily obscuration of the sky in the images. To prevent birds from perching on the TSI, ecologically friendly "bird spikes" were 


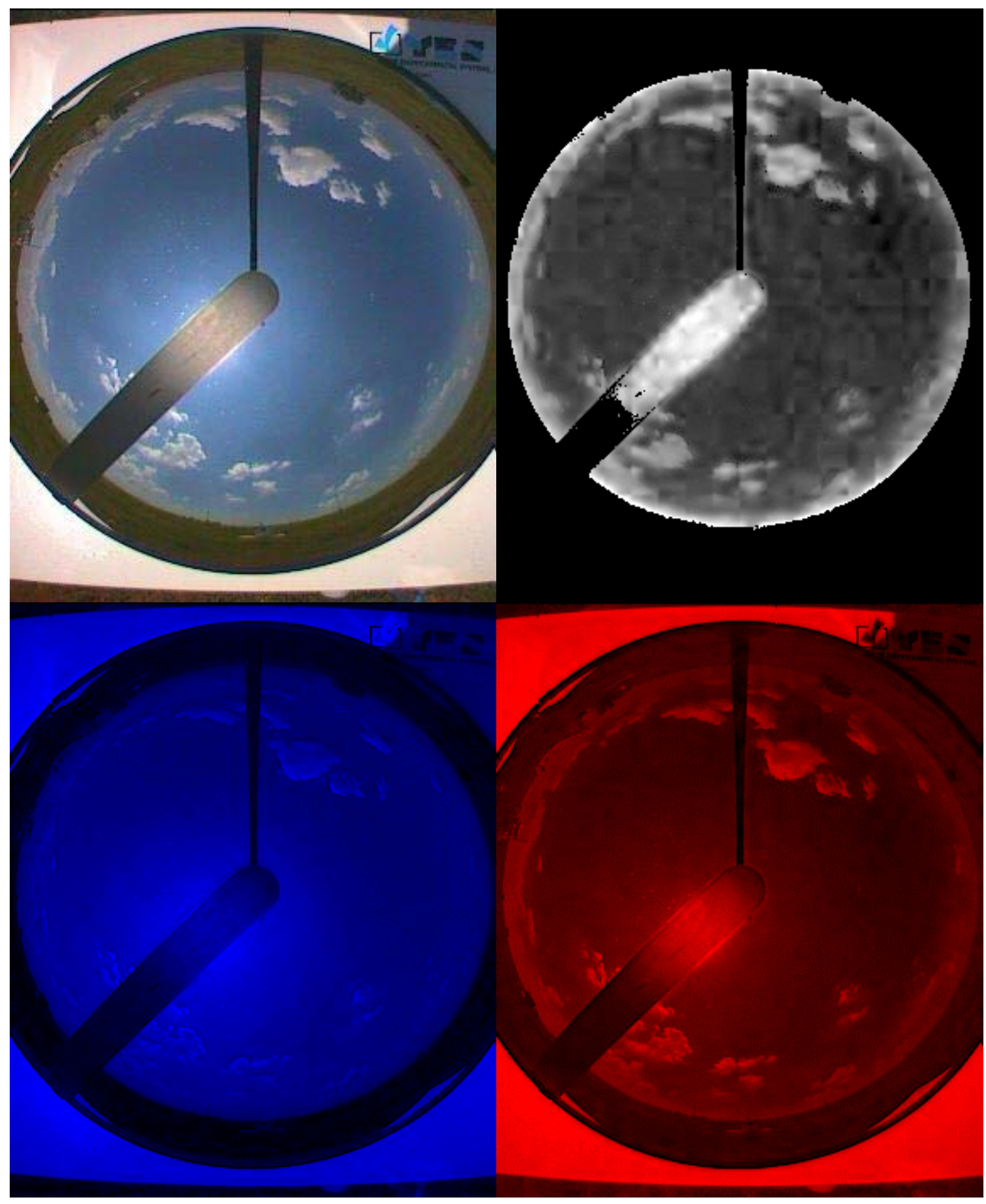

Figure 4. Cloudy-Sky Image (upper left), Corresponding Relative Red/Blue Ratio "Image" (upper right), Separated Blue (lower left), and Red (lower right) Pixel Value Amount Images. 


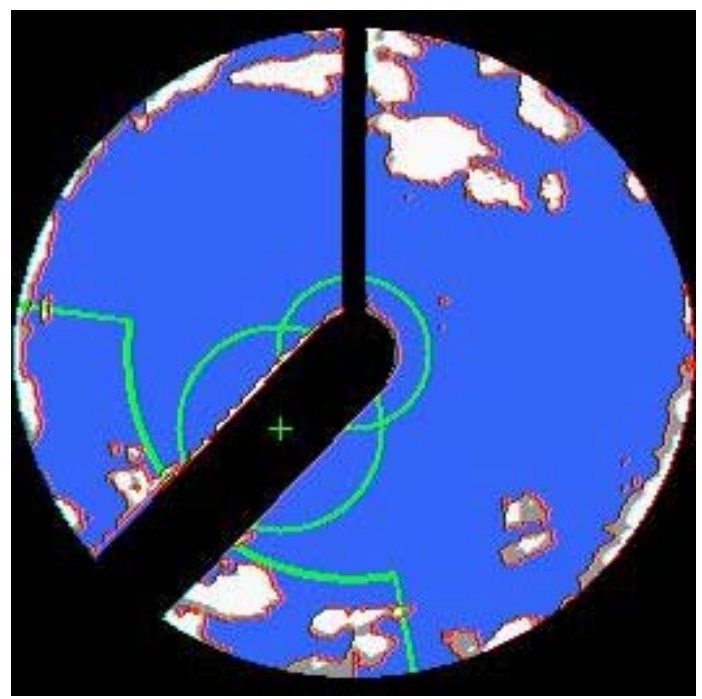

Figure 5. Cloud Decision Image Corresponding to Figure 4. Black denotes masking. Green outlines denote areas where separate cloud/clear pixel counts are included in the sky cover retrieval output files. Cloud decision color scheme is clear (blue), thin cloud (gray) and opaque cloud (white).

installed on the horizontal portions of the camera housing and camera arm assembly. These plastic spike assemblies are passive, do not pollute the surroundings either acoustically or chemically, and merely prevent the birds from being able to land on these surfaces, thus do not cause injury. Since the spikes were installed, no instances of bird droppings on the TSI mirror have been observed.

\section{Testing and Evaluation Results}

After the 2000 Cloud IOP/ARESE II (ARM Enhanced Shortwave Experiment II) deployment, the TSI 880 was deployed at the ARM SGP CF for system testing and evaluation. The original plan called for a deployment of 6 months to a year to test system reliability, and to establish a good seasonal statistical record for a TSI - WSI (Whole Sky Imager) comparison. The TSI 880 was installed within about 50 feet of the SGP CF WSI, as shown in Figure 6, to facilitate direct comparison of sky images and sky cover retrievals between the two systems.

\subsection{System Reliability}

Data are gathered from the TSI 880 by a PC operating the Yankee YESDAQ software and located at PNNL. This YESDAQ software is scheduled to automatically gather and add to the database the latest data via Internet connection every 15 minutes during daylight hours. For the 1-minute rate, the TSI-880 on-board "hot" storage, i.e., that storage area automatically accessible by the YESDAQ software via Internet, can store up to about 2 days worth of data before it is overwritten by newer data. The on-board 


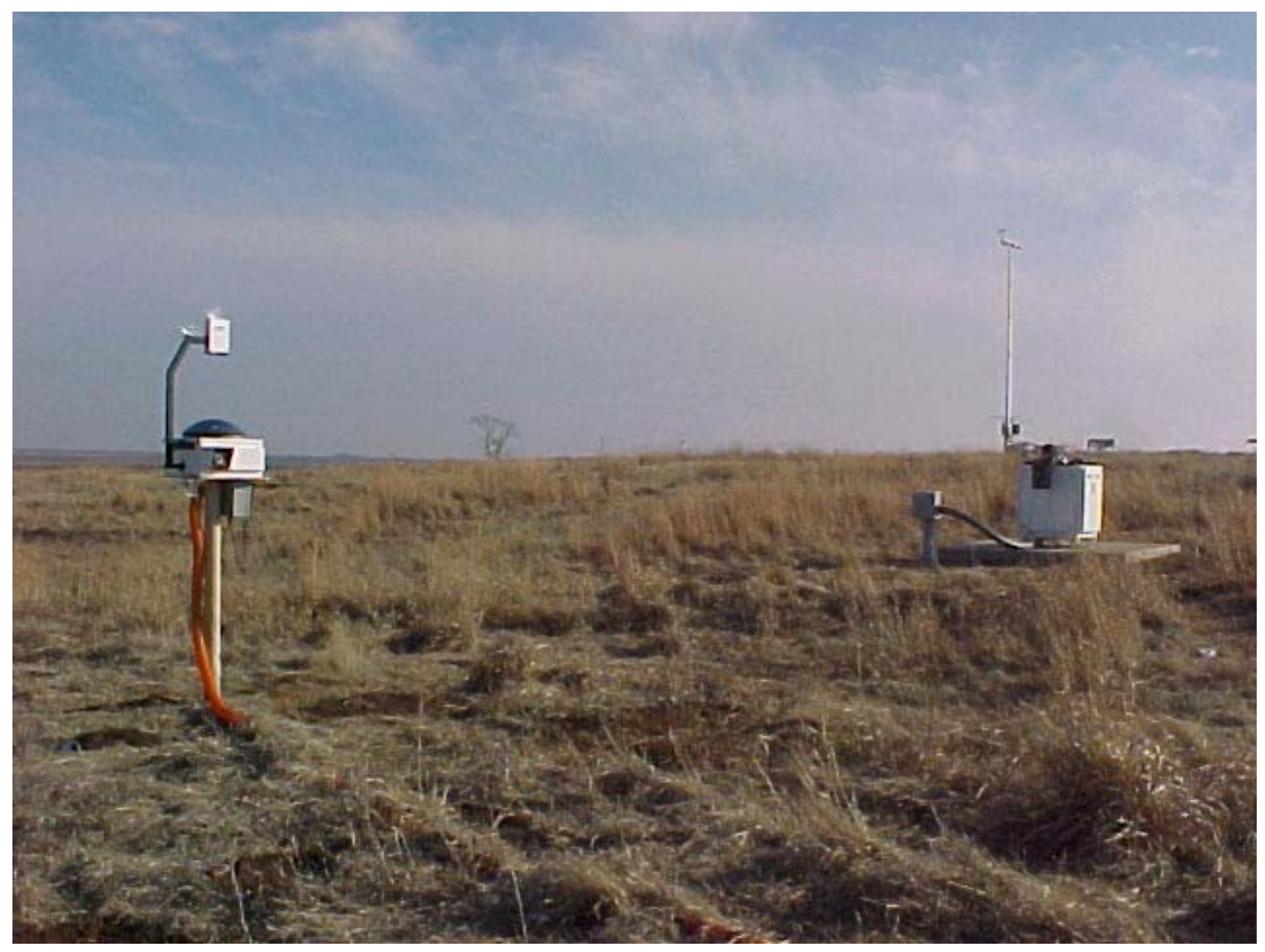

Figure 6. Picture Showing the Relative Closeness of the TSI (left) and WSI (right) at the SGP.

Data Storage Module can store about 2 weeks worth of data that are available in the form of bundled tar files, which can be manually gathered via Web browser. Thus, data that are not gathered automatically for some reason (power or Internet failure, etc.) can still be manually ingested into the YESDAQ up to two weeks later.

Figure 7 shows the number of daylight retrievals of fractional sky cover per day that were attained (red line) for the first 6 months of operation at the SGP, as well as the number possible (blue line, solar zenith angle $\leq 85^{\circ}$ ). The TSI was installed on July 1,2000 , but the data collection didn't start until late on this day. The large loss of data that occurred on September 17 and 18 was due to operator travel, the automated data gathering being missed due to a PC problem at PNNL, while the system operator was away on extended travel. By the time the operator returned, these two days of data had already been overwritten on the Data Storage Module and were unrecoverable. The slight change in possible data shown on about August 14, 2000, is due to a setting change, where the solar elevation limit for processing of images to infer sky cover was changed from the factory default of $3^{\circ}$ above the horizon to $5^{\circ}$. Figure 8 shows the same data as in Figure 7, but here as the ratio of the actual divided by the possible data. The line plotted then is in effect the percent of possible data. As is shown, not including the July 1, 2000, startup day but including all else, on average about 98 percent of the possible data were collected. Thus, for this period of testing, the TSI has proven to be a reliable operational system. 


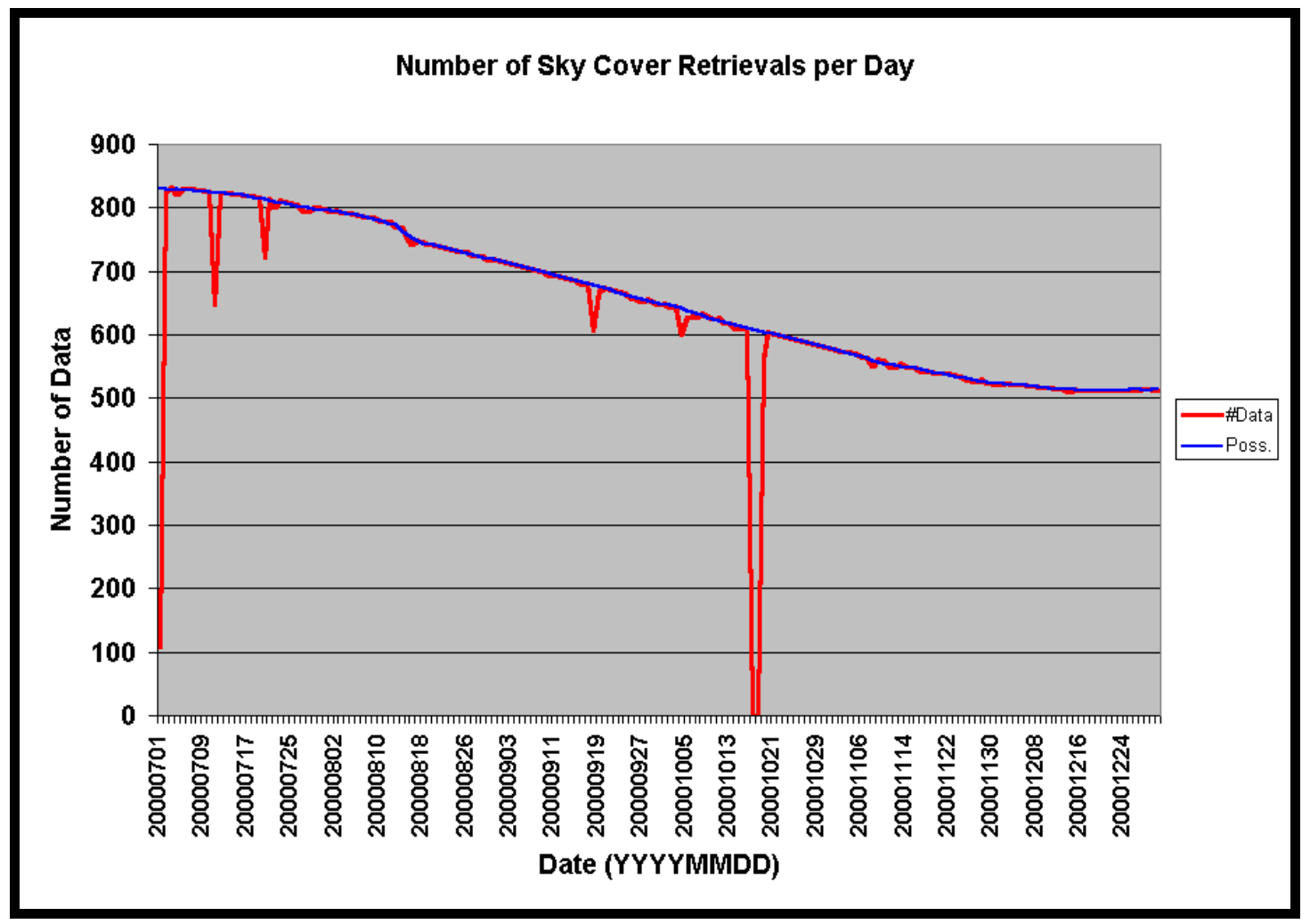

Figure 7. Plot of the Actual (red) and Possible (blue) Number of TSI Daylight Retrievals of Fractional Sky Cover at the SGP from July 1 through December 31, 2000.

\subsection{Comparison of Sky Cover Retrievals}

\section{Background}

During last year's ARESE II IOP, both the ARM Whole Sky Imager (WSI) and the new TSI model 880 were operated simultaneously in close proximity to one another. Both instruments were located at the BTA for the duration of this IOP. Sky cover estimates from these systems during the interval March 9, 2001 to April 6, 2001, have been compared to evaluate the agreement of these two fully independent systems. A primary purpose of the present comparison is to provide a link between the large volume of WSI cloud data gathered within the ARM Program and the increasing usage of TSI instruments by other researchers and research programs, as well as ARM (the TSI has recently been accepted as an official ARM instrument). A secondary purpose is to provide assurance of the sky cover estimates for the upcoming Island Effect Study to take place in 2001 to 2002 on Nauru Island. During this effort, a TSI will be deployed on the windward side of the island and operated in concert with an additional TSI placed near the WSI at the Denig site to study the effects of the Nauru landmass on measurements at the Denig site. 


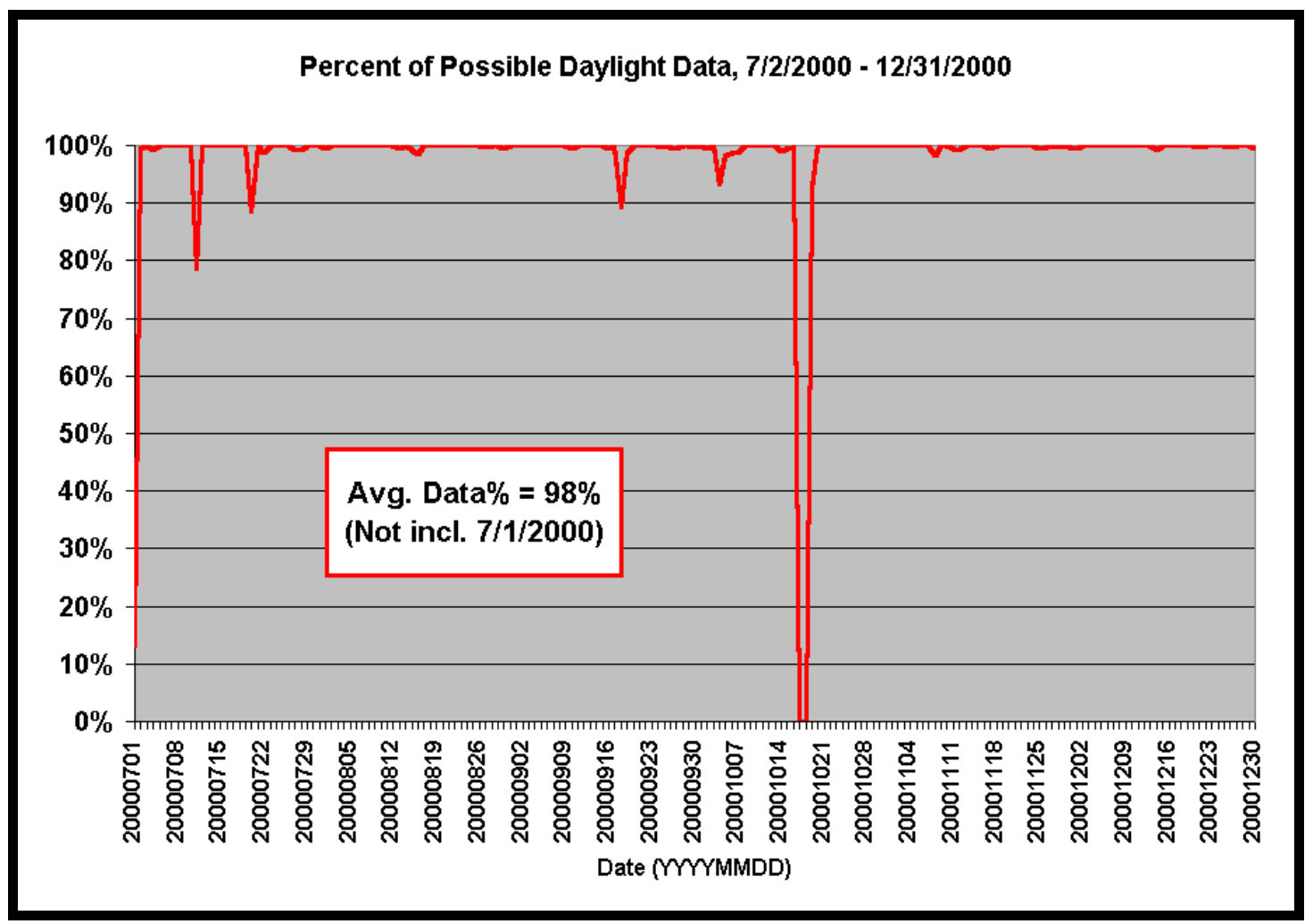

Figure 8. Plot of the Actual Divided by the Possible Number of TSI Daylight Retrievals of Fractional Sky Cover at the SGP from July 1 through December 31, 2000.

\section{Procedure for TSI/WSI Sky Cover Comparisons}

The TSI has a 1-minute repeat time for acquiring images while the WSI was operated with a 6-minute repeat interval during ARESE II, necessitating time synchronization. All time differences were found to be less than 5 seconds. Where the TSI reports sky cover fraction for the entire $160^{\circ}$ field-of-view, the WSI reports sky cover in 8 sectors of the sky: zenith to $45^{\circ}$, and $45^{\circ}$ to $80^{\circ}$ zenith angle for each of the $\mathrm{N} / \mathrm{E} / \mathrm{S} / \mathrm{W}$ directional quadrants. Each of the sky cover amounts reported by the WSI in these quadrants were weighted, dependent on that sectors total projected sky area onto the image, and summed with the others to yield a percentage of the pixels that are categorized as a particular cloud type. In addition, the 'indeterminate' and 'undefined' portion of each sector are added and used to modify the sky cover estimates to approximate the percentage that would be assessed were obstacles not in the field of view or were there not areas whose color ratio exceeds those allowed by the WSI processing software. For the WSI, the sky classifications summed to represent total sky cover were the 'bright,' 'intermediate,' and 'dark' cloud classifications. The 'aerosol' classification by the WSI was not included in this work because of the large uncertainty of the distinction between aerosol and thin cloud. For the TSI, the sum of 'thin' and 'opaque' classifications was used for comparison. Initially, comparisons were made using the preliminary data available, yielding approximately 2500 coincidences. The results reported here consist of comparisons made using the more quality-controlled, reprocessed data from both instruments, which 
produced approximately 2000 coincidences. The decrease in coincidences is primarily from loss of WSI retrievals, though the count was also affected by restricting cloud estimates to intervals when the solar elevation angle exceeded $10^{\circ}$ due to increased uncertainties for low sun angles.

\section{TSI/WSI Comparison Results}

The total number of TSI - WSI coincidences acquired during the March 9 through April 6, 2001, interval amounted to 2,004 pairs of sky cover estimates. A comparison of corresponding sky cover values is shown in Figure 9, which shows the 'total' sky cover of the WSI versus both the 'total' and 'opaque' of the TSI processing. The TSI appears to routinely produce slightly higher total sky cover estimates than the WSI. This, and the comparison of WSI total sky cover to only the TSI 'opaque' retrievals, suggests that the primary difference between the TSI and WSI retrievals lies in the specific limits of clear/thin and thin/opaque set for each system. The WSI processing yields two sky classifications that were not included in sums used for this study: 'aerosol' and 'mixed aerosol and cloud,' the latter category not being currently populated by the ARM Program WSI processing. Several examples of the corresponding total sky cover estimates made by the TSI and WSI are presented in Figures 10 through 12. These data represent all available coincidences of the processed data (those data remaining after quality control procedures) for the 3 days shown and when the solar elevation angle exceeds $10^{\circ}$. Certainly the temporal behavior is quite consistent between the WSI and TSI derived sky cover, as are the sky cover values themselves. Again, there does appear to be a tendency for TSI total sky cover estimates to slightly exceed those from the WSI, a common feature in the comparisons that we attribute to slightly different thresholds applied in the processing to detect cloud presence.

TSI vs WSI Cloud Cover (Total)

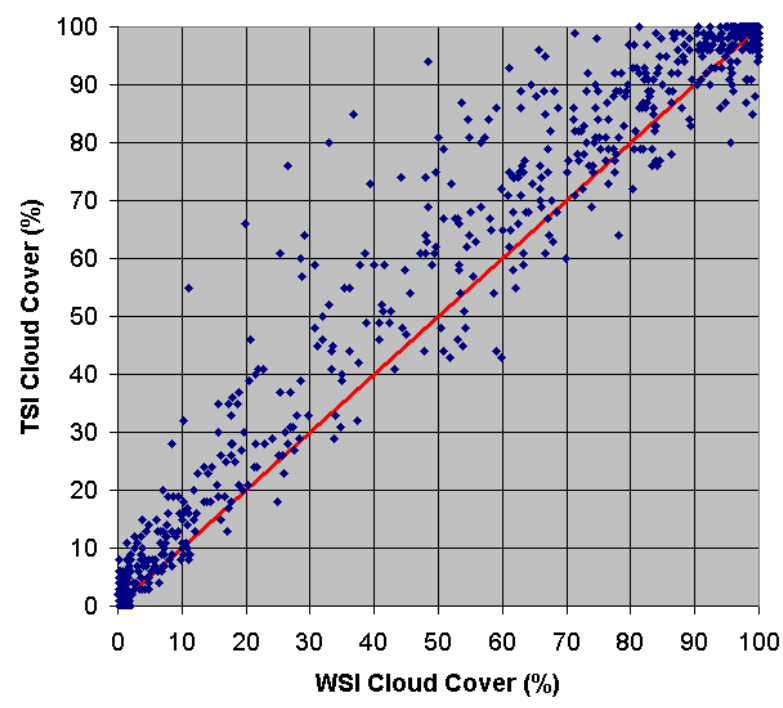

WSI vs TSI Cloud Cover (Opaque)

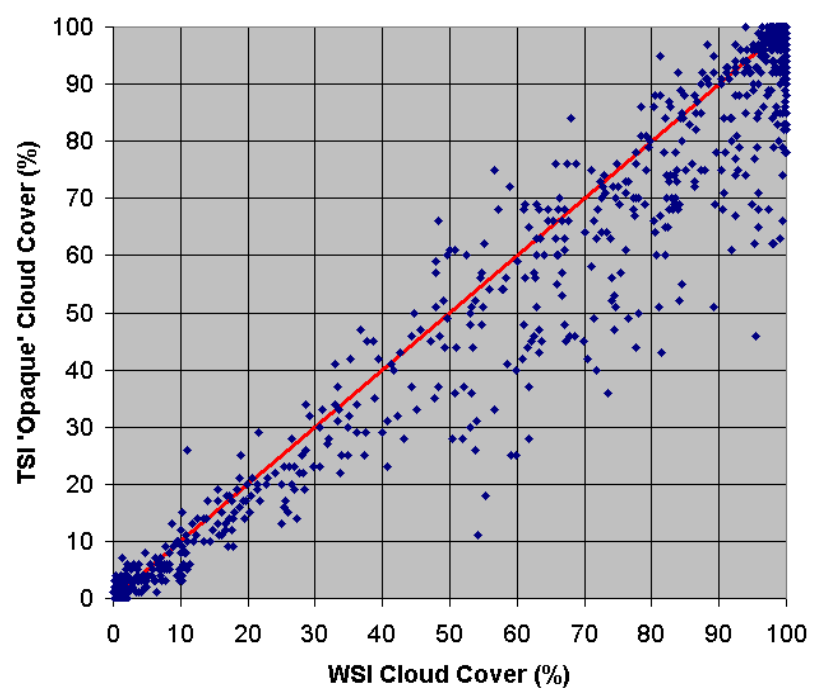

Figure 9. Plot of WSI Sky Cover (X axis) Versus TSI Total (left) and Opaque (right) Sky Cover. 
March 12, 2000 TSI and WSI Total Sky Cover (\%)

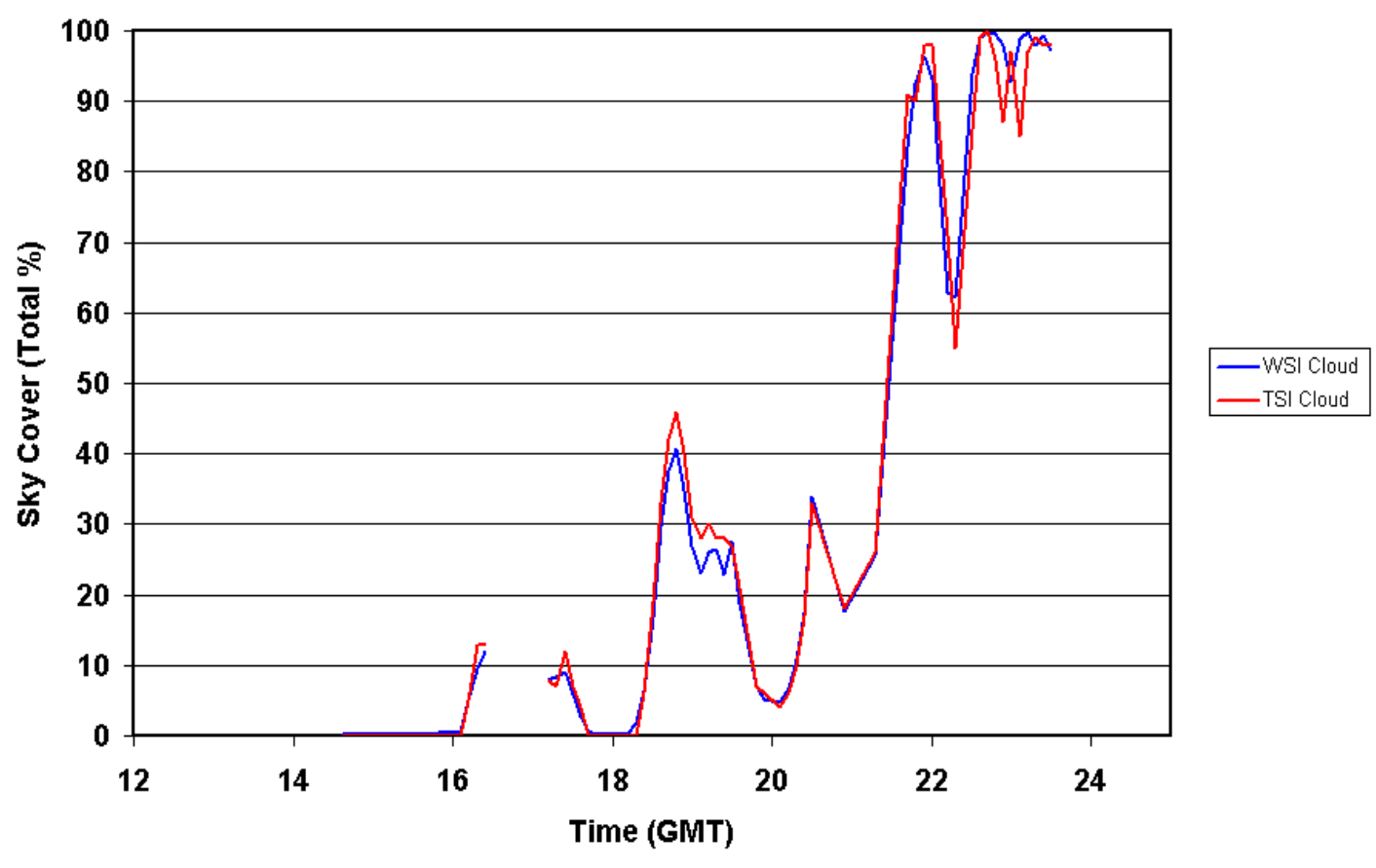

Figure 10. Time Series of WSI (blue) and TSI Total (red) Total Sky Cover for March 12, 2000.

In order to identify any obvious trends of WSI - TSI sky cover differences, we have plotted these differences as functions of TSI 'total' cloud cover (Figure 13), TSI 'thin' cloud cover (Figure 14), and GMT (Figure 15). There appears to be a slight trend towards an increased TSI estimate of cloud cover relative to the WSI during partially cloudy conditions, though there is also increased scatter of the differences as compared to both fully cloudy and clear conditions (Figure 13). This is most likely the result of the process for estimating 'thin' cloud conditions as evidenced in Figure 14, where the magnitude of the WSI - TSI difference tends to increase with TSI 'thin' cloud amounts. The magnitude of differences seems to have no correlation with time of day, as shown in Figure 15.

Regardless of the slight bias between the TSI and WSI total sky cover retrievals, there is in the aggregate a surprising level of agreement. We say surprising because in fact these two sky imaging systems were completely independently developed, and the operational limits for cloud classification were completely independently set for clear/cloud discrimination by the individual system operators. Figure 16 shows the difference in percent of 'total' sky cover for the entire set of 2,004 coincident observations in terms of the number (left axis) and frequency of occurrence (right axis). Analysis of these results shows that 87 percent of the WSI - TSI total sky cover percent differences fall within \pm 5 percent, with agreement within \pm 10 percent occurring 94 percent of the time. Thus, even given the nebulous definition of what is and is not a cloud in the atmospheric sciences, these two systems show excellent agreement. 
March 30, 2000 TSI and WSI Total Sky Cover (\%)

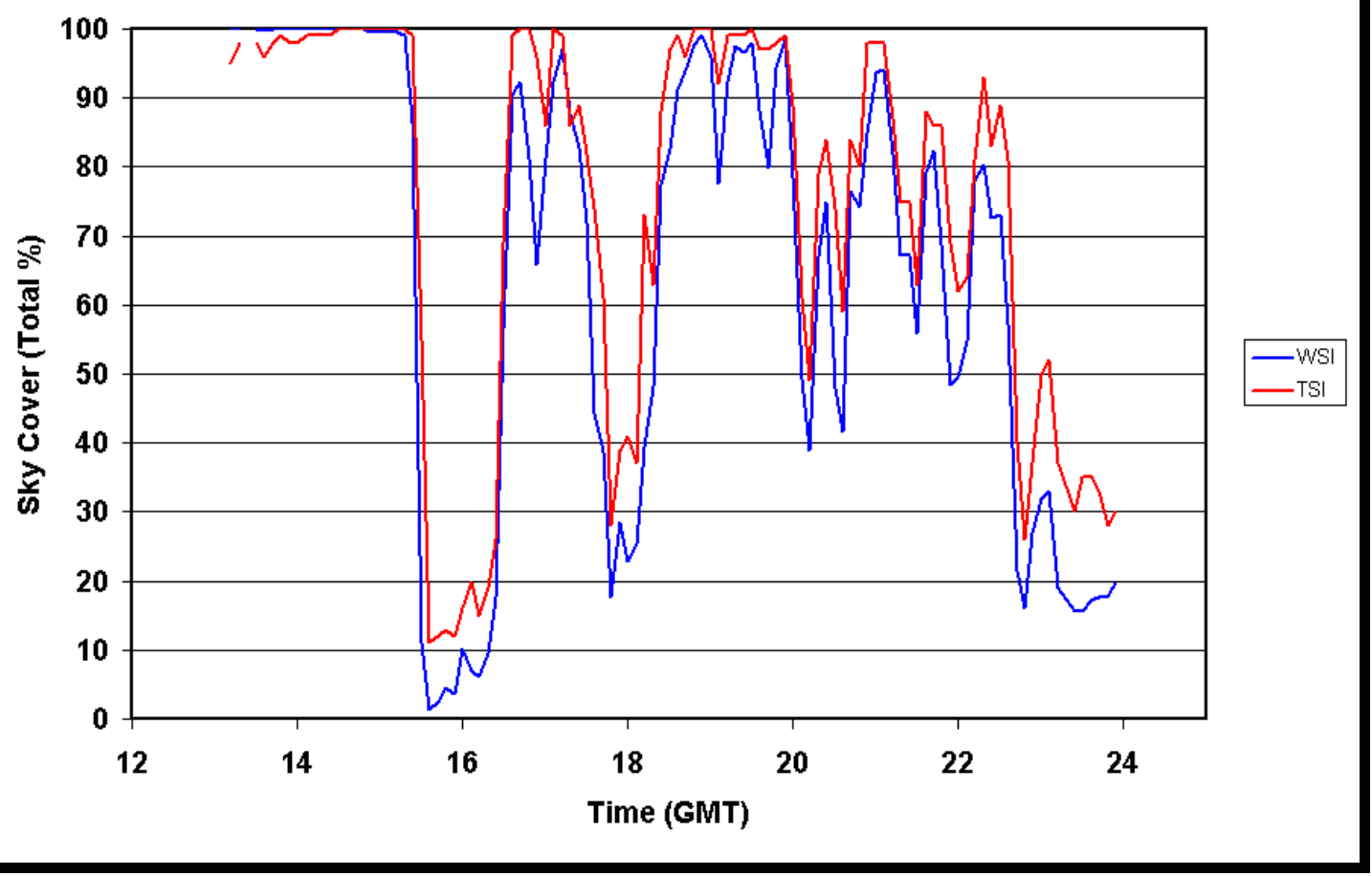

Figure 11. Same as Figure 10, but for March 12, 2000.

\section{Summary of Comparison Results}

1. Comparison of sky cover estimates from the WSI and TSI using independent methods of analysis illustrates that a consistent estimate of sky cover can be routinely generated with current analysis techniques.

2. The TSI does favor a slightly higher total sky cover estimate than the WSI, which we attribute primarily to the user-defined threshold that identifies the 'thin' cloud classification. The decision process by which one defines aerosol or very thin cloud is not necessarily unique. We speculate that population of the WSI 'mixed aerosol and cloud' category by ARM WSI data processing might shift the comparison toward better agreement.

3. The probability of agreement between the TSI and WSI to within 5 percent sky cover is quite high at 87 percent. The probability of agreement for clear and fully cloudy conditions is nearly 100 percent, falling off somewhat for partially cloudy conditions when thin clouds or aerosol are prominent.

4. Judging from the total number of observations during this nearly month long period and the number of final sky cover estimates, the WSI seems to have a somewhat more restrictive quality control mechanism. Further comparisons will be made with the much larger data archives available since the deployment of a TSI to the SGP CF on July 1, 2000 in close proximity to the ARM WSI. 


\section{April 03, 2000 TSI and WSI Total Sky Cover (\%)}

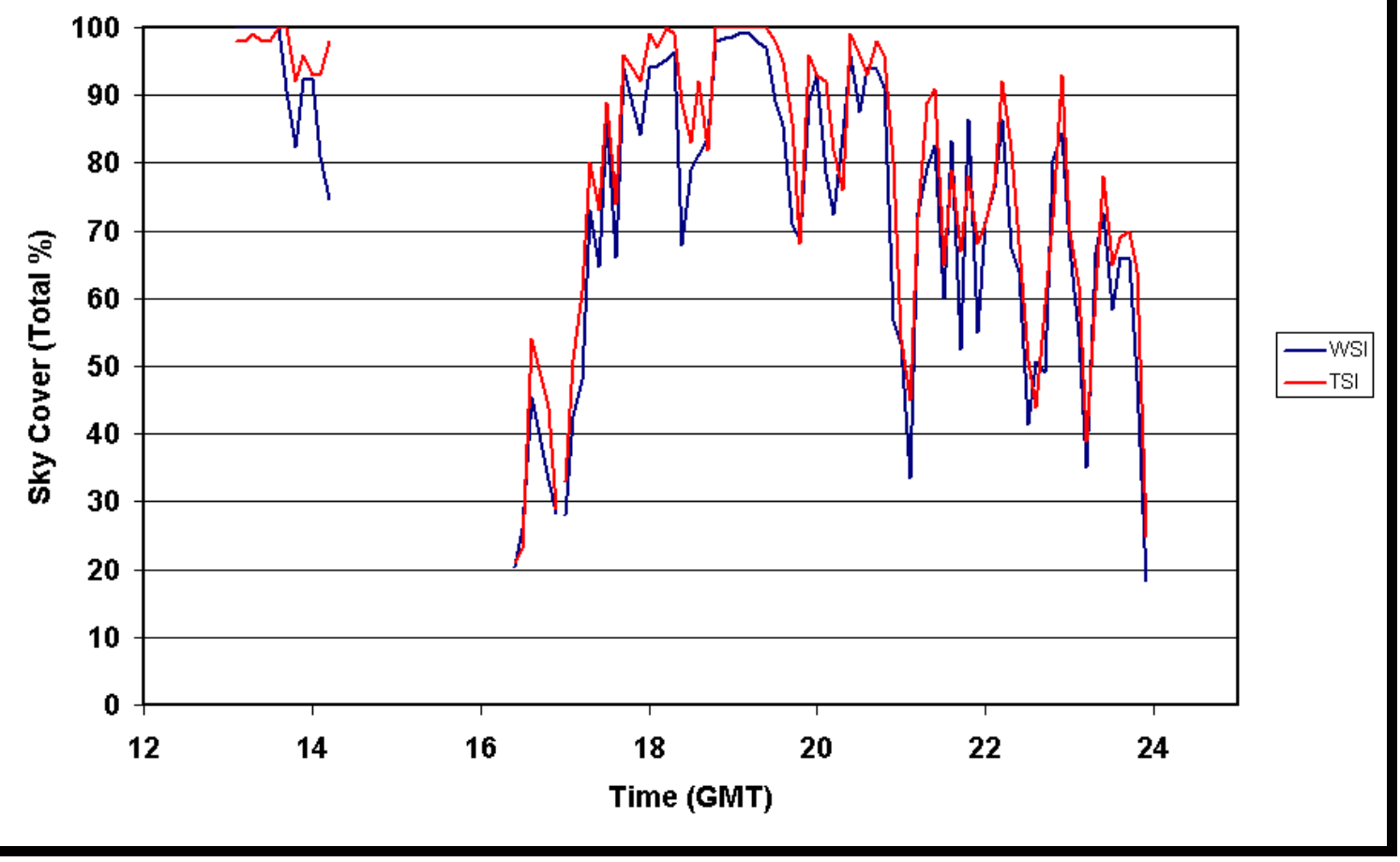

Figure 12. Same as Figure 10, but for April 3, 2000.

\section{Summary}

The testing and evaluation to date has shown no inherent problems with the TSI 880 system that would preclude use as a long-term deployment field instrument. Onsite daily maintenance requirements include only cleaning of the TSI mirror, similar to the daily radiometer dome cleaning currently in practice in ARM, for best results. Testing during the prototype development of the Hemispheric Sky Imager shows that the mirror design is less susceptible to debris influence on sky cover retrievals than the smaller dome surface area of upward facing camera systems (Long et al. 1998). Thus, the TSI can also generally produce good results in situations such as those at the ARM SGP Extended Facilities, where maintenance visits occur on a bi-weekly basis. The data return rate of 98 percent, even given operator errors, is fairly high. The agreement shown here with sky cover retrievals from a co-located WSI suggests that routine measurements of this type are well within the realm of acceptable repeatability limits. Naturally, since there is no common agreement on what constitutes cloud versus clear and thus no absolute standard, the question of absolute accuracy cannot be answered.

We are currently waiting processing of the ARM SGP WSI data from July, 2000 to present in order to continue the TSI - WSI comparison. The originally agreed upon 1-year testing and evaluation period extends through June 30, 2001. After that time, a complete long-term investigation of seasonal agreement will be undertaken, and the long-term comparison results will be presented as an addition to this current work. 


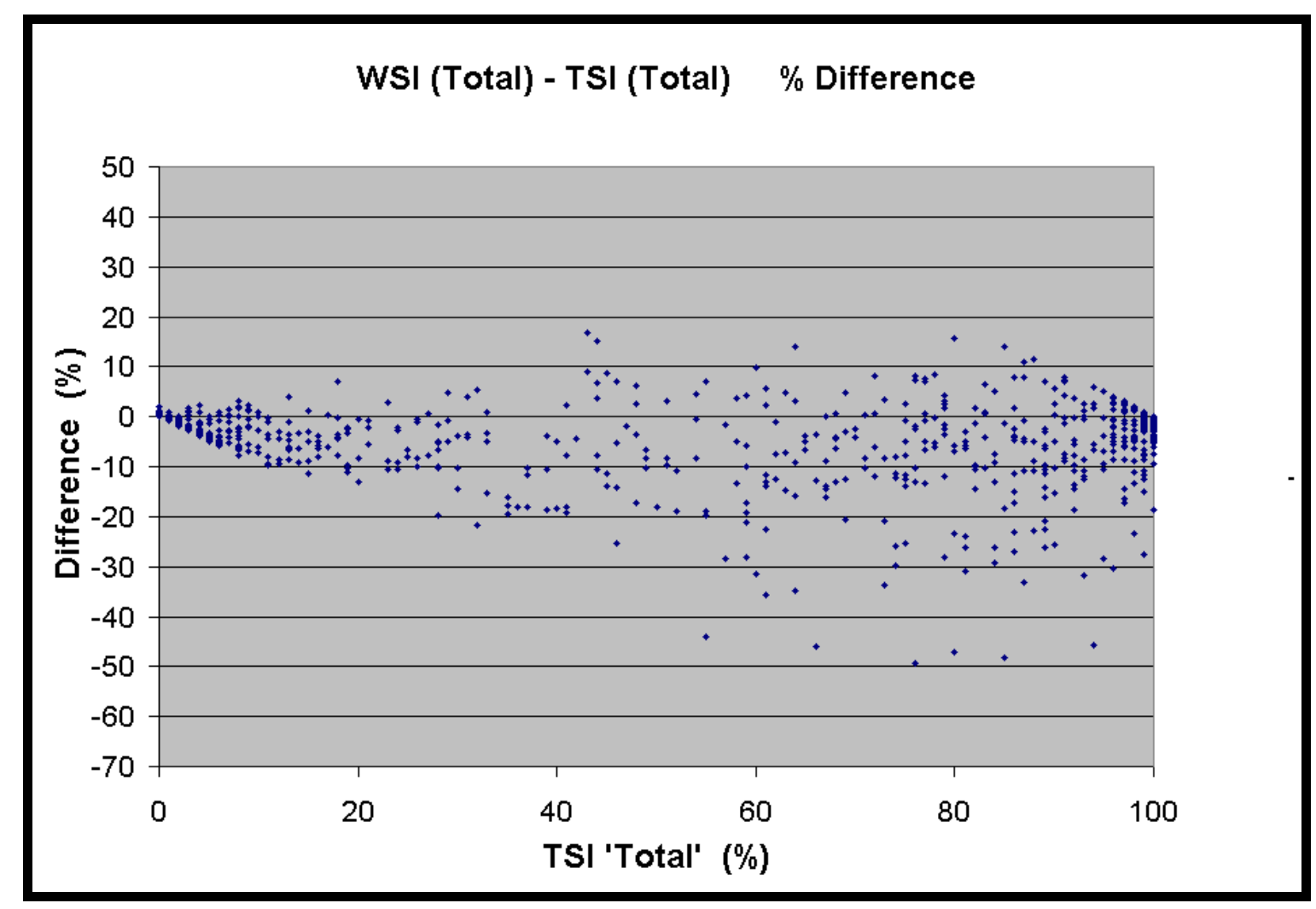

Figure 13. Plot of WSI - TSI Total Sky Cover Difference Versus TSI Total Sky Cover Amount.

\section{References}

Long, C. N. and J. J. DeLuisi. 1998: Development of an Automated Hemispheric Sky Imager for Cloud Fraction Retrievals. In Proc. 10th Symp. on Meteorological Observations and Instrumentation, January 11 to 16, 1998, Phoenix, Arizona. 


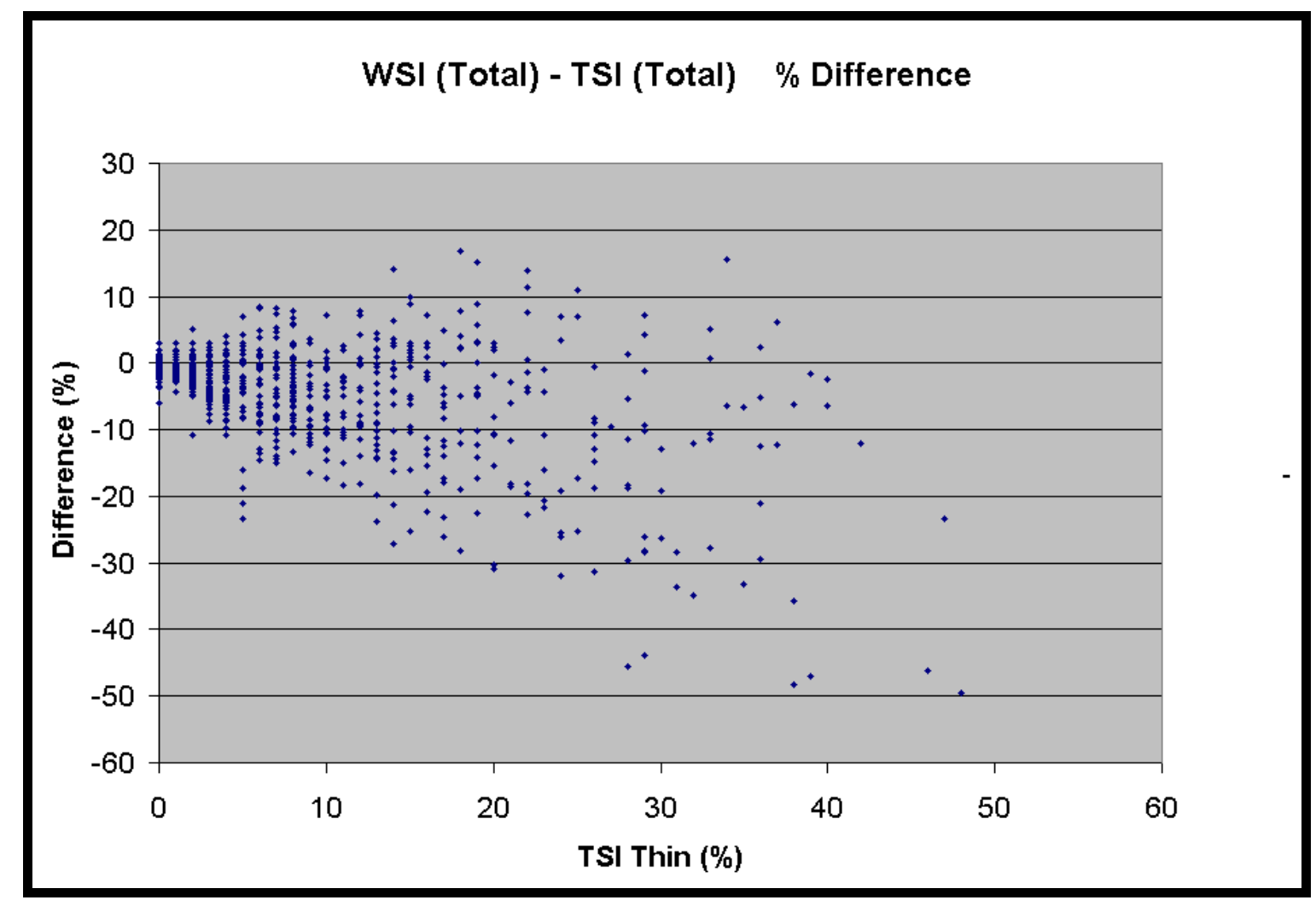

Figure 14. Plot of WSI - TSI Total Sky Cover Difference Versus TSI Thin Sky Cover Amount. 


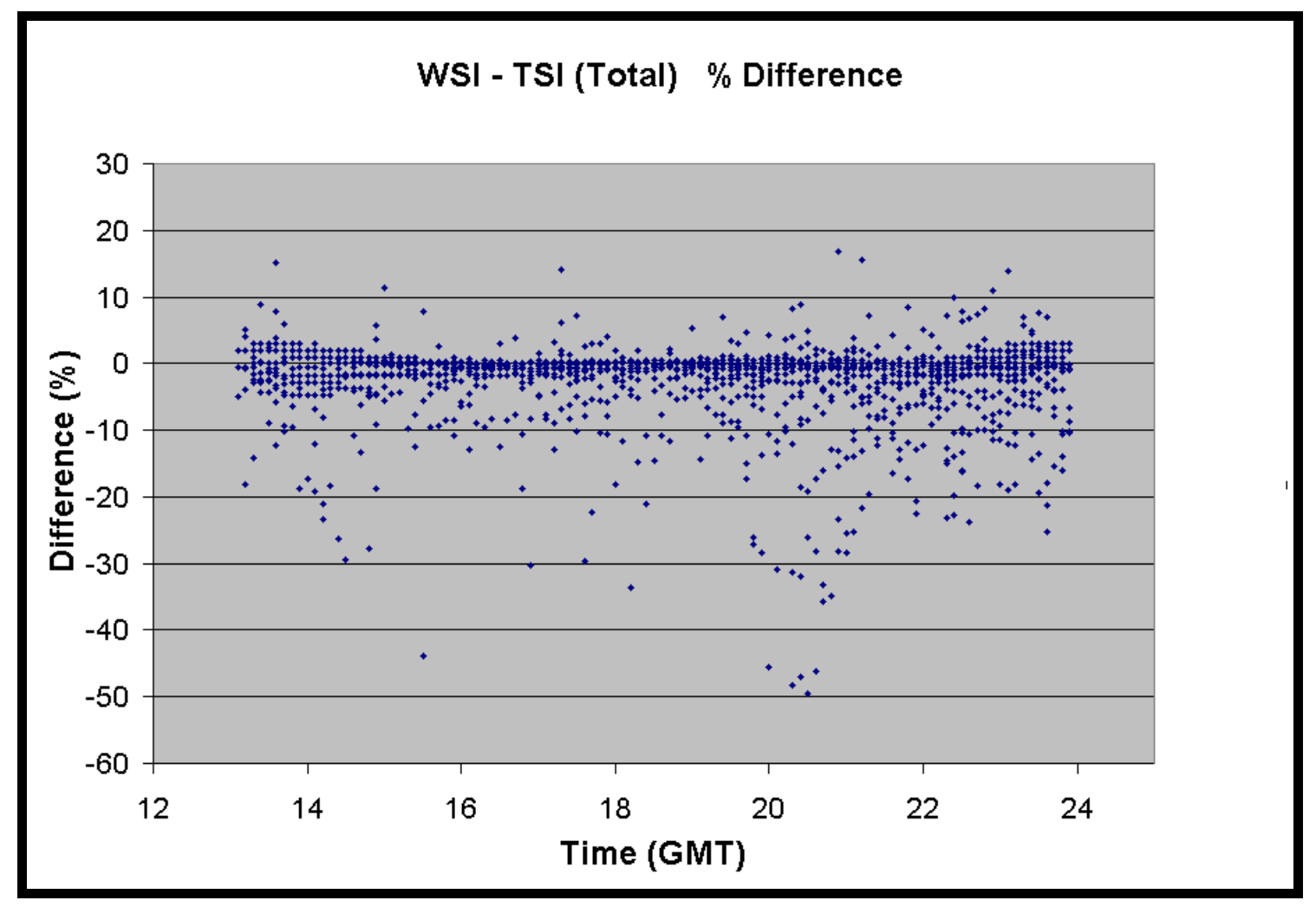

Figure 15. Plot of WSI - TSI Total Sky Cover Difference Versus Time of Day. 


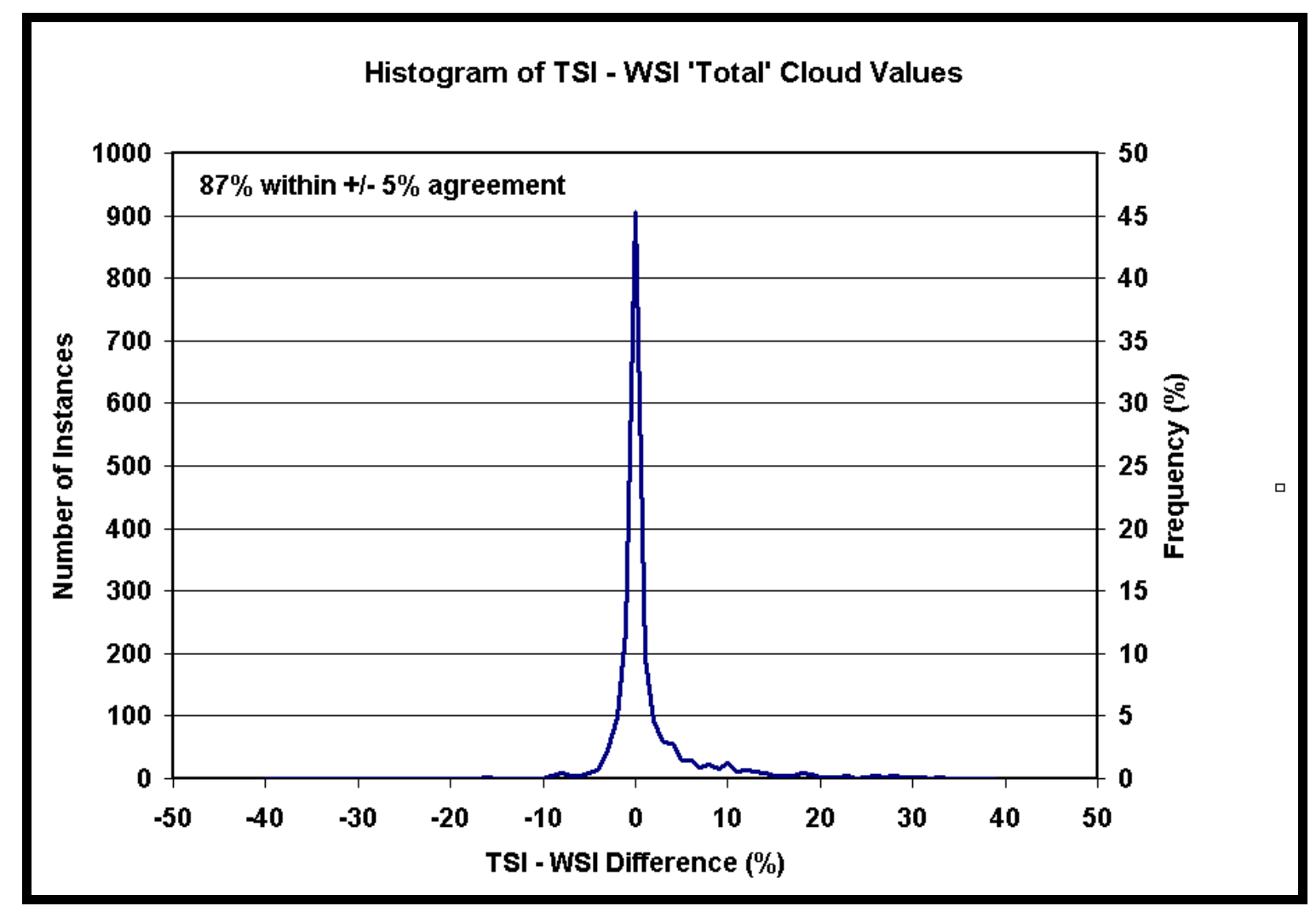

Figure 16. Histogram of WSI - TSI Sky Cover Percent Difference for this Study in Terms of Number of Instances (left axis) and Relative Frequency (right axis). 


\section{Appendix A}

\section{TSI 880 Operating Specifications}




\section{Appendix A}

\section{TSI 880 Operating Specifications}

The TSI- 880 has the following operating specifications:

\begin{tabular}{|c|c|}
\hline CHARACTERISTIC & SPECIFICATION \\
\hline Imager resolution & $352 \times 288,24$-bit color JPEG format \\
\hline Sampling rate & $\begin{array}{l}\text { Variable, with a maximum of one image every } 15 \\
\text { seconds. }\end{array}$ \\
\hline $\begin{array}{l}\text { Operating } \\
\text { temperature }\end{array}$ & $-40^{\circ} \mathrm{C}$ to $44^{\circ} \mathrm{C}$ \\
\hline Weight & Approximately $70 \mathrm{lbs}(32 \mathrm{~kg})$ \\
\hline Dimensions & $\begin{array}{l}\text { Base: Approximately } 16 \mathrm{in} . \times 14 \mathrm{in} .(41 \mathrm{~cm} \mathrm{x} 36 \mathrm{~cm}) \\
\text { Height: Approximately } 28 \mathrm{in} .(71 \mathrm{~cm}) \text { to top of } \\
\text { support arm }\end{array}$ \\
\hline Power requirements & $\begin{array}{l}110 / 220 \text { VAC } \\
\text { Heater duty cycle varies with air temperature: } \\
\text { approximately } 500 \mathrm{~W} \text { with heater on; } 50 \mathrm{~W} \text { with heater } \\
\text { off. }\end{array}$ \\
\hline Software & $\begin{array}{l}\text { Supports 32-bit Web browsers including Netscape } \\
\text { Navigator or Microsoft }{ }^{\mathbb{B}} \text { Internet Explorer (versions } 4 \\
\text { or higher) on Windows }{ }^{18} 9 x / \mathrm{NT} \text {, Macintosh } \\
\text { UNIX. }\end{array}$ \\
\hline Data telemetry & $\begin{array}{l}\text { Modem or TCP/IP via Internet. An optional Data } \\
\text { Storage Module (DSM) is available for remote } \\
\text { standalone sites. }\end{array}$ \\
\hline
\end{tabular}




\section{Appendix B}

TSI Operation and Monitoring Features 


\section{Appendix B}

\section{TSI Operation and Monitoring Features}

The TSI can be set up, operated, and monitored remotely by direct Web based connection to the system. For monitoring current performance, a user connects to the TSI 880 by entering the TSI 880 Web address into a browser. The browser then displays the most recent image in a choice of 4 different formats, the "Panorama" example is shown in Figure B.1. This same Web interface allows the system operator to set and/or change all aspects of the TSI 880 operations and processing. Figure B2 shows an example screen for adjusting the image center, masking of the sun blocking strip, and enabling and adjusting the sizes of the various "special pixel count areas" for sky cover retrieval. Figure B3 shows an example of the set up screen for adjusting and testing the clear/thin and thin/opaque cloud detection threshold limits, and the "sun meter" limit.

In addition to the above connections directly to the TSI 880 itself for real time monitoring, the system stores data in a database that can be used for monitoring the record, and assessment of quality of the sky cover retrievals. This Web based interface is called the "Data Visualization Engine" by the manufacturer, and in our case can be accessed by the community at http://armtsil.pnl.gov. This link will produce the main page shown in Figure B4. The system operator uses this interface for system settings such as scheduling data collection jobs, data management, and reprocessing. The community user uses this main page to access and view the data. The user clicks on the "All Sites and Instruments" link in the left panel, which brings up the sites page as shown in Figure B5. Clicking on a site, such as "ARMSGP," will bring up a list of instruments at that site, in this case just "TSI-880". Clicking on "TSI-880" then brings up the instrument's page, as shown in Figure B6.

Once connected to an instrument's page, various forms of data visualization are presented, with the exception of choosing the year. By choosing a month, a graphical display of the daily percent of time the sun was not obscured by cloud is presented, i.e., a daily "sunshine meter." This plot is useful to distinguish between days, for instance a high sun percent value indicates a sunny day, where a low sun percent value indicates a cloudy day, and a middle sun percent value indicates a partly cloudy day. This can serve to discriminate between types of days that might be of interest to the user.

Clicking on a day brings up a fractional sky cover plot, a sample of which is shown in Figure B7. This plot shows the time series of retrieved opaque and thin sky cover amounts. In addition, there is an "MPEG" button located below the plot legend on the right. Clicking this button will generate an mpeg movie from the day's sky images. This movie is useful for quick viewing of the sky for the entire day to locate times and/or cloud cover situations of interest. The user can then click on individual times, or 
ranges of times, or use one of the pull-down choices to sub-sample the day's images, as shown in Figure B8 displaying every 100th sample. These Web pages described here were used for the TSI 880 evaluation and testing to operate and monitor the instrument deployed at the SGP CF.

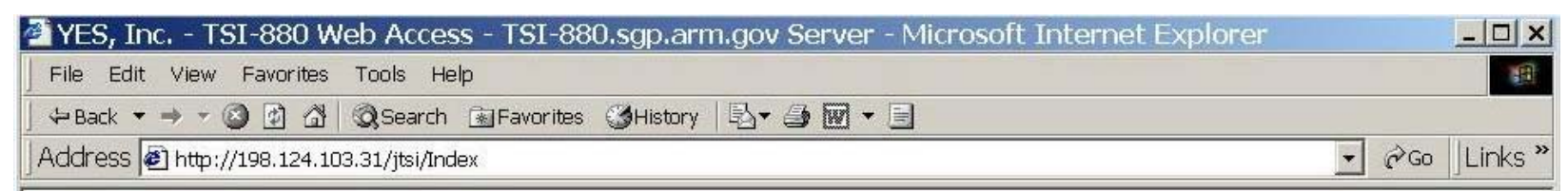

\section{TSI-880 Total Sky Imager}

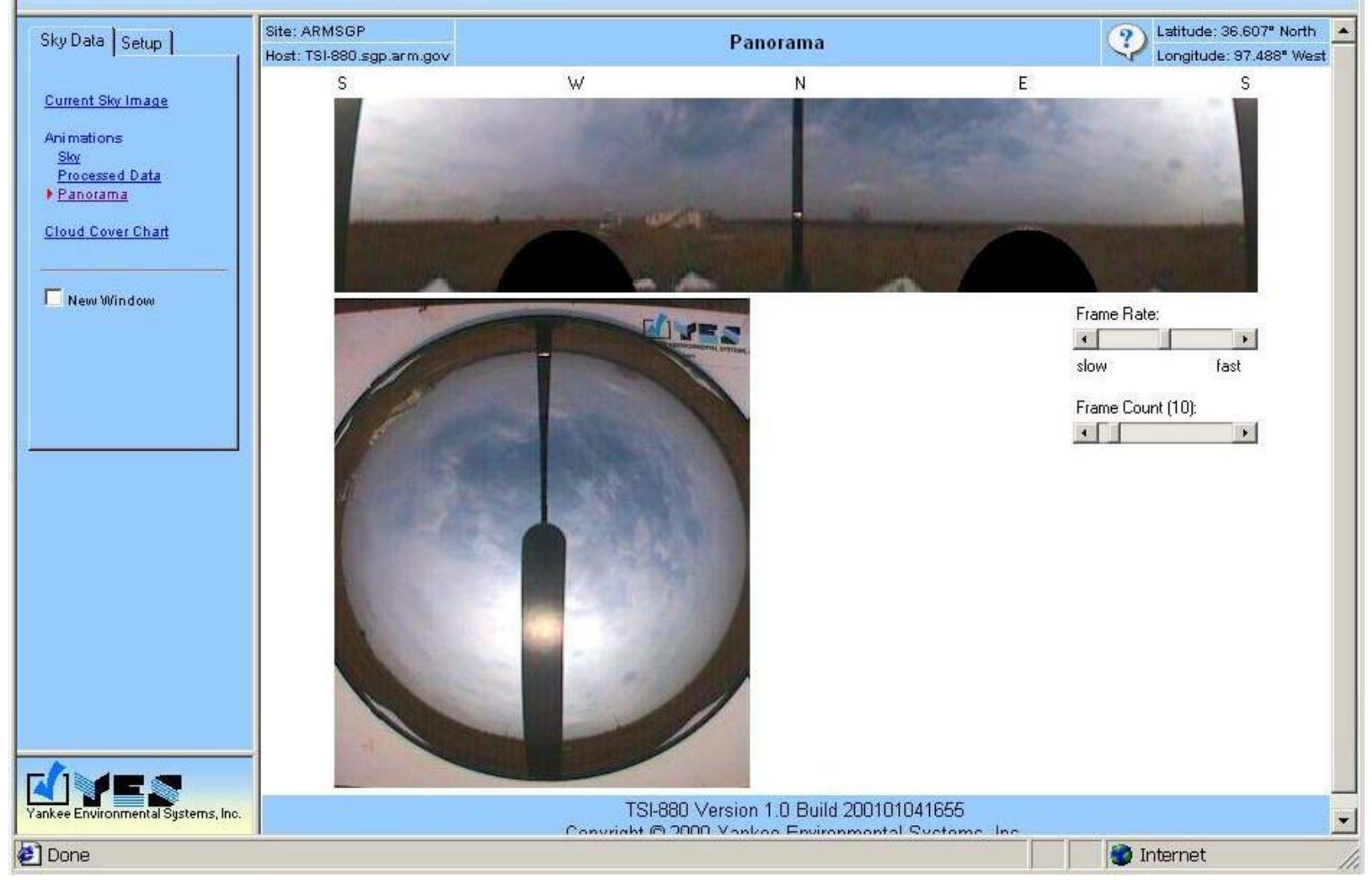

Figure B1. Sample of the "Panorama" Screen for Monitoring Current Performance of the TSI 880. 


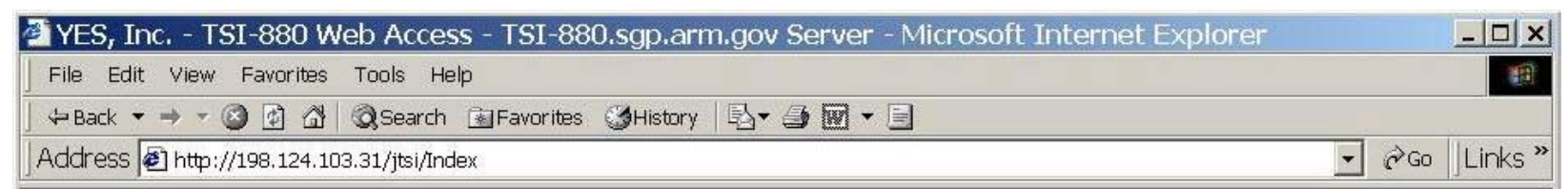

\section{TSI-880 Total Sky Imager}

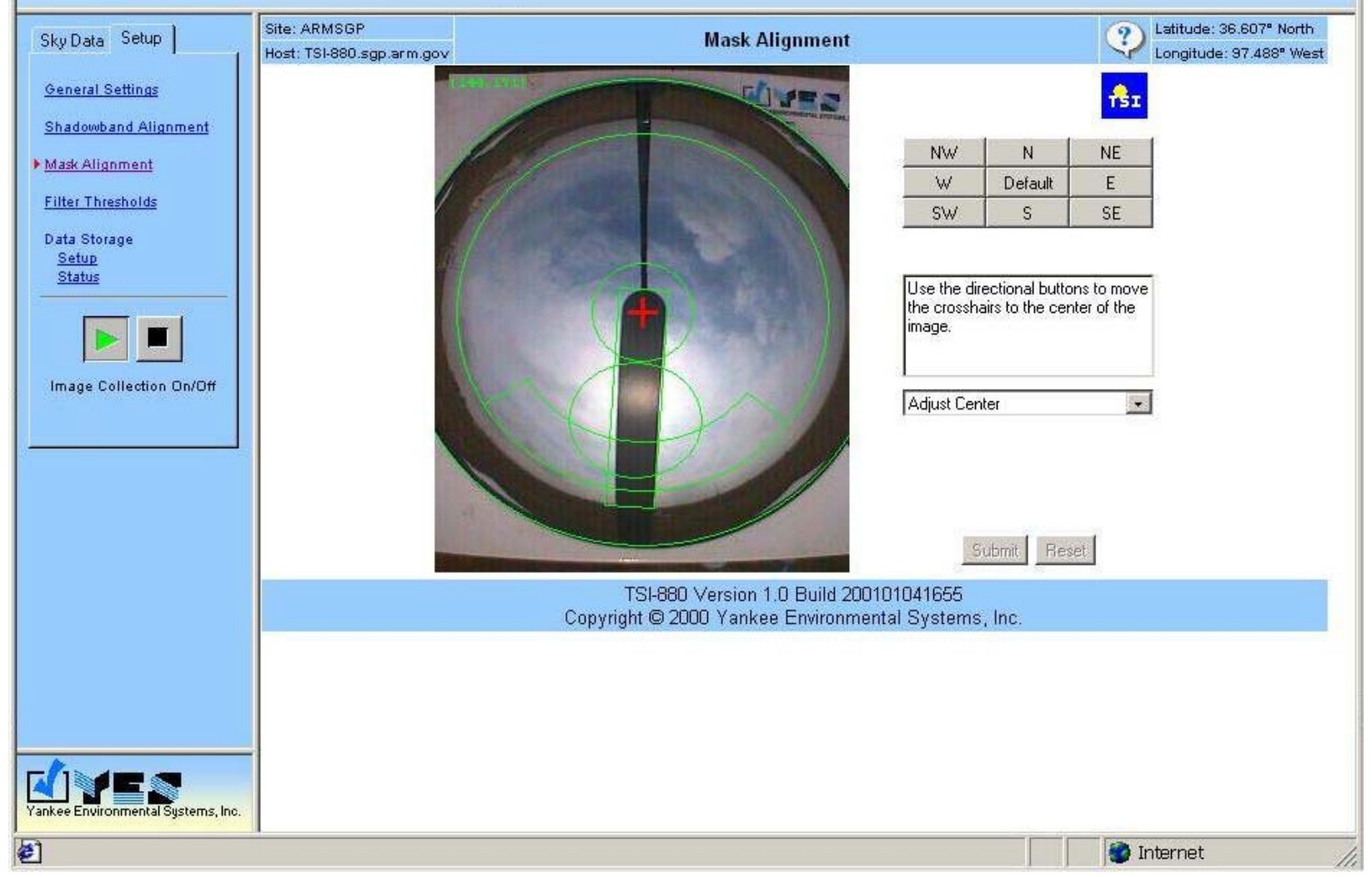

Figure B2. Sample of the TSI 880 Set Up Screen For Adjusting Image Centering, Masking, and Special Area Sizes. 


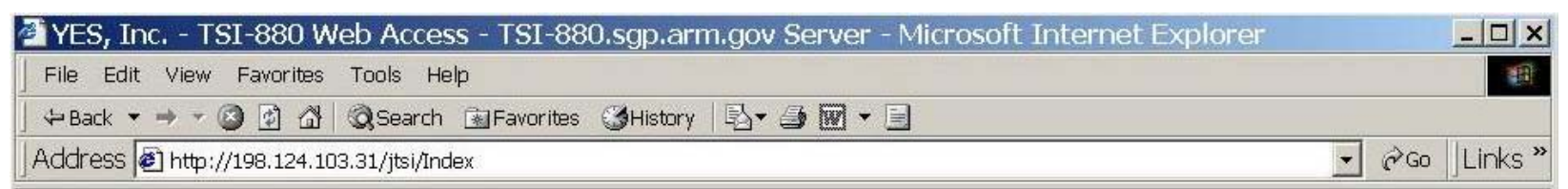

\section{TSI-880 Total Sky Imager}

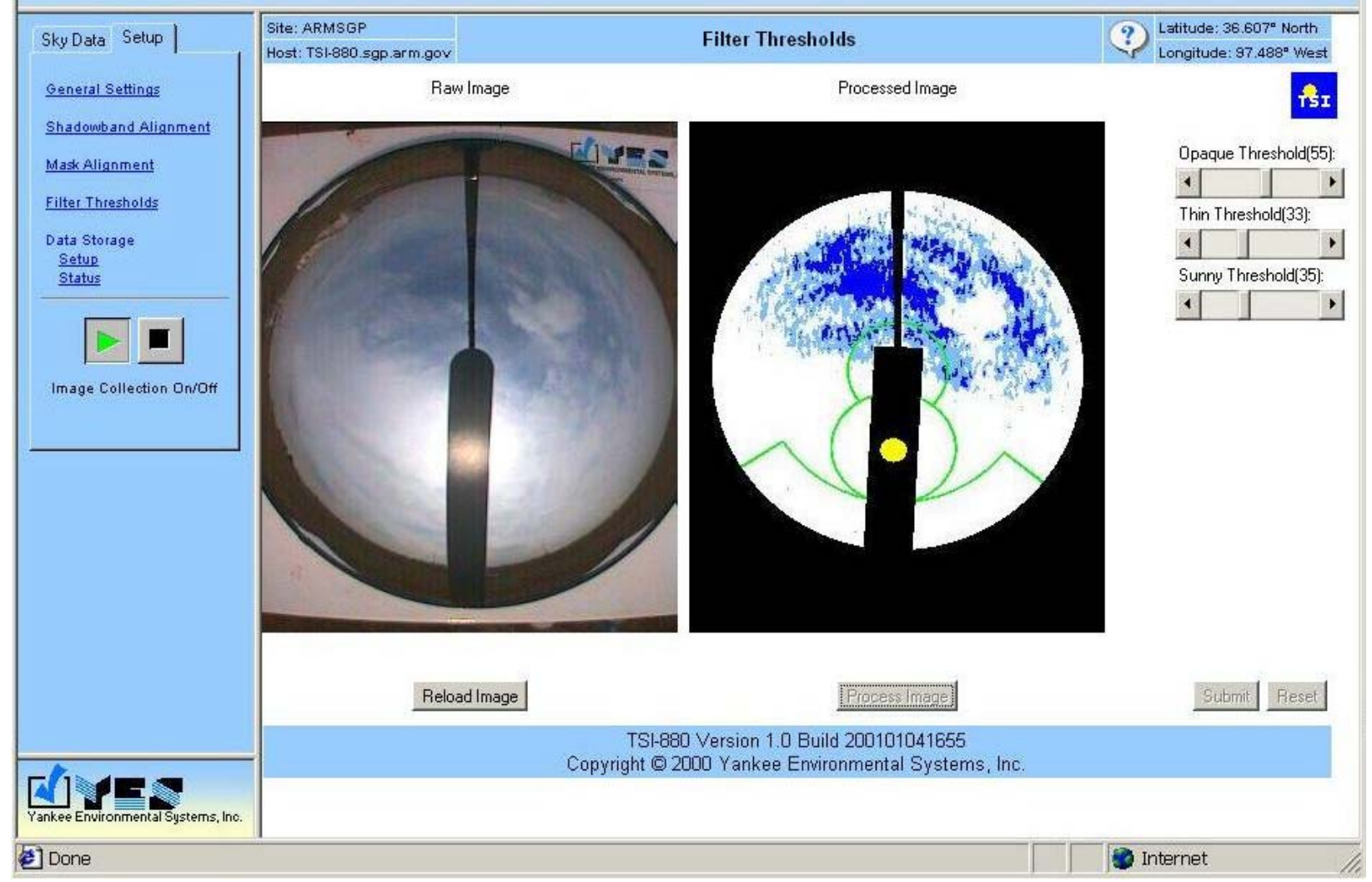

Figure B3. Sample of the TSI 880 Set Up Screen for Adjusting and Testing the Clear/Thin and Thin/Opaque Cloud Detection Limits, and the "Sun Meter" Threshold. 


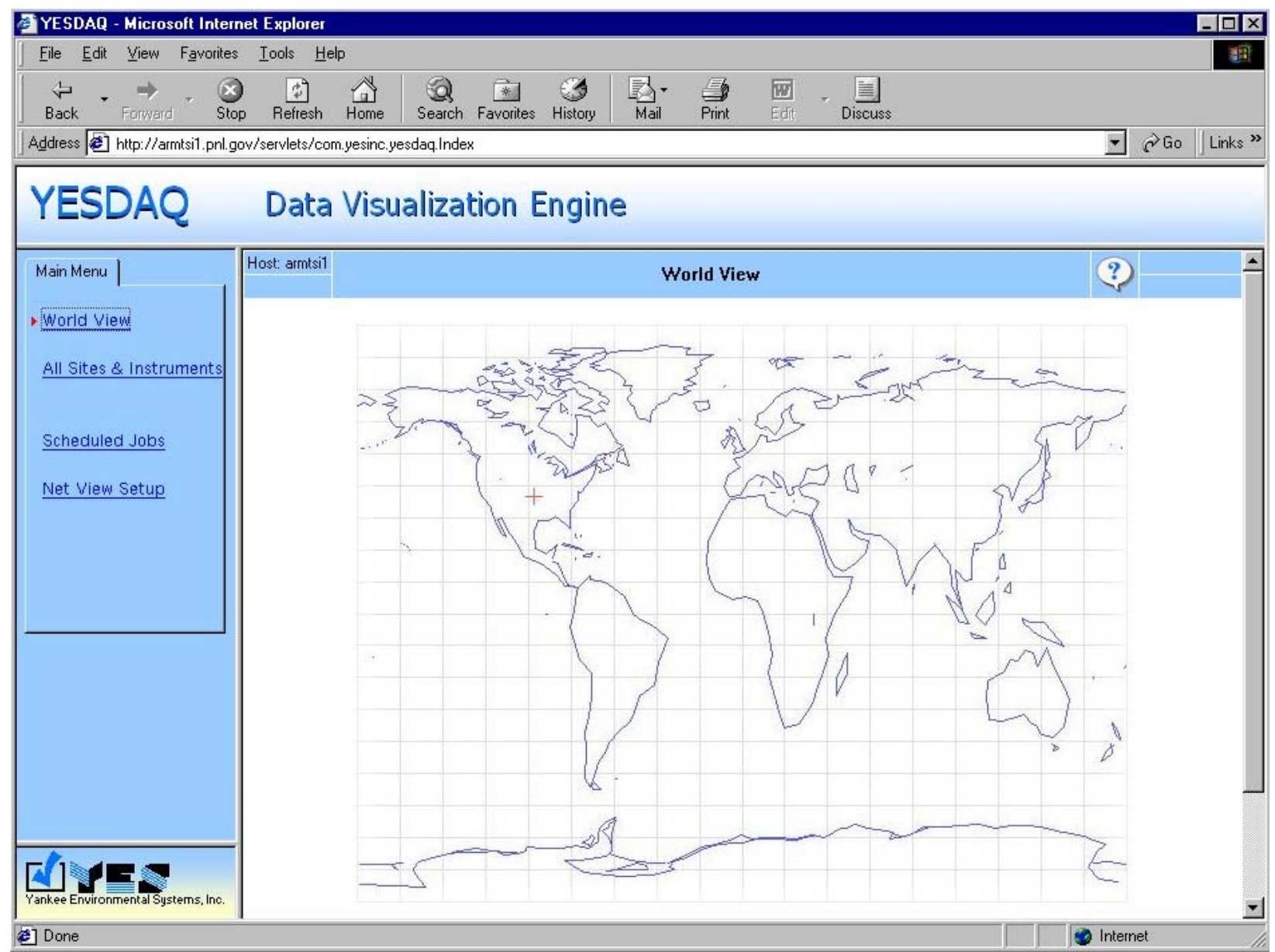

Figure B4. Sample of the TSI 880 Data Visualization Engine Main Page. 


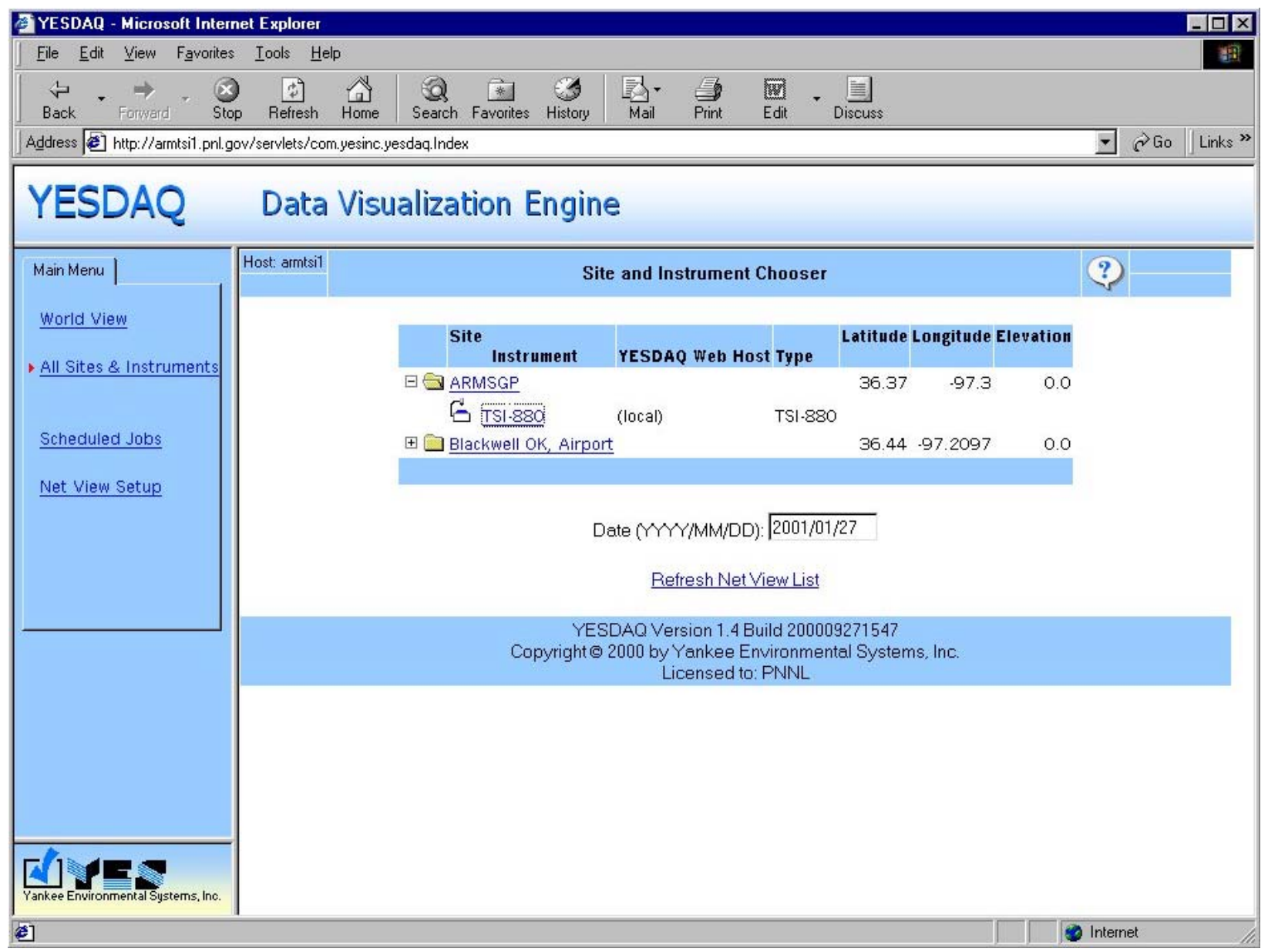

Figure B5. Sample of the TSI 880 Data Visualization Engine Sites Page. 
E TSI-880@ARMSGP - Microsoft Internet Explorer

Eile Édit View Fạvorites Iools Help

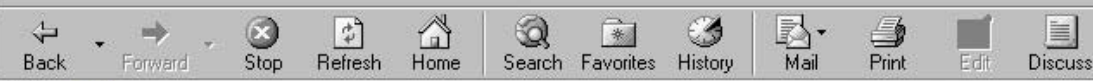

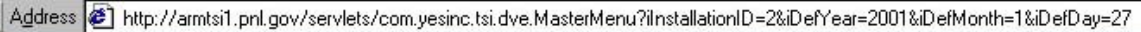

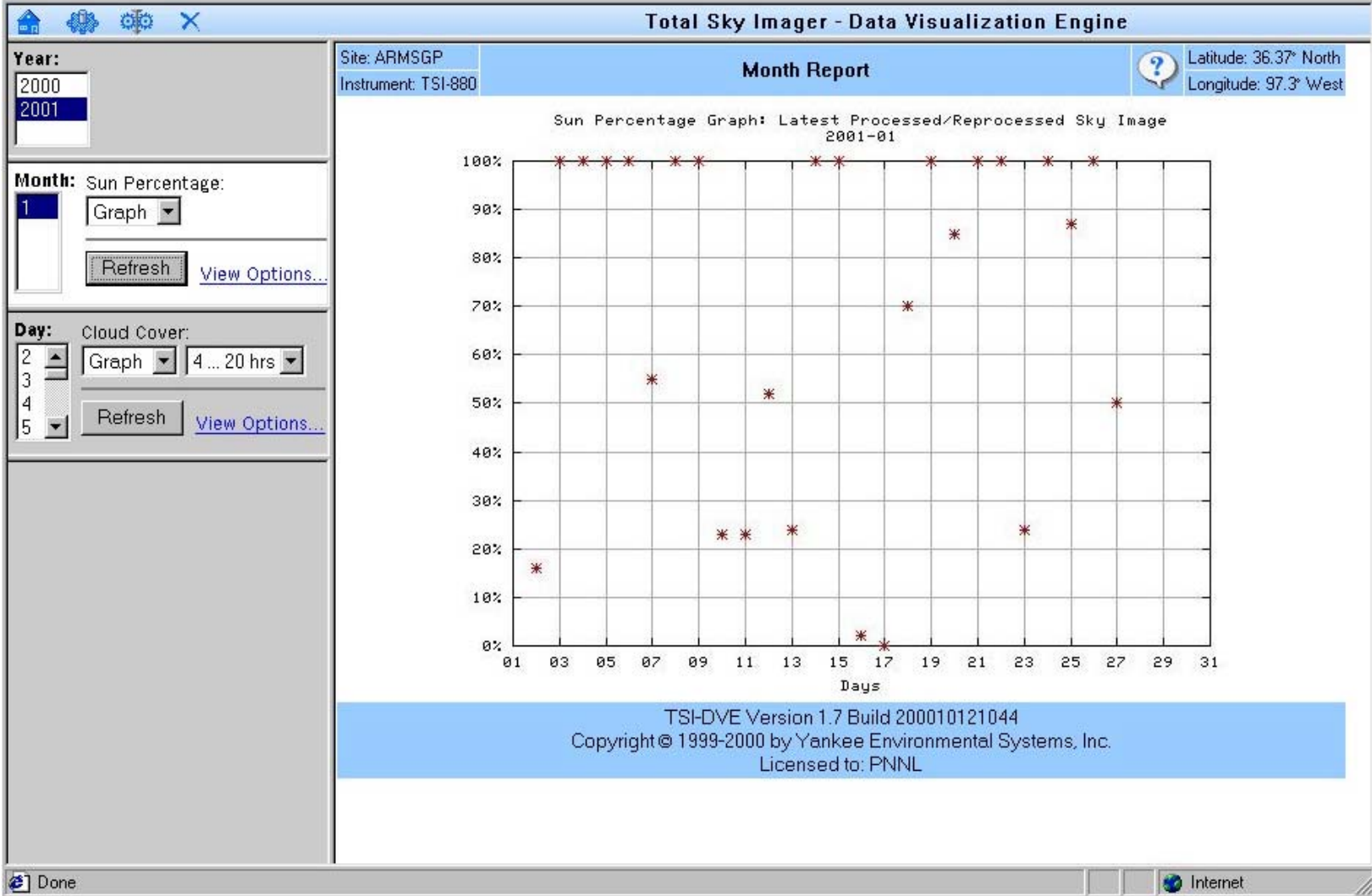

Figure B6. Sample of the TSI 880 Data Visualization Engine Daily Sun Meter Page. 


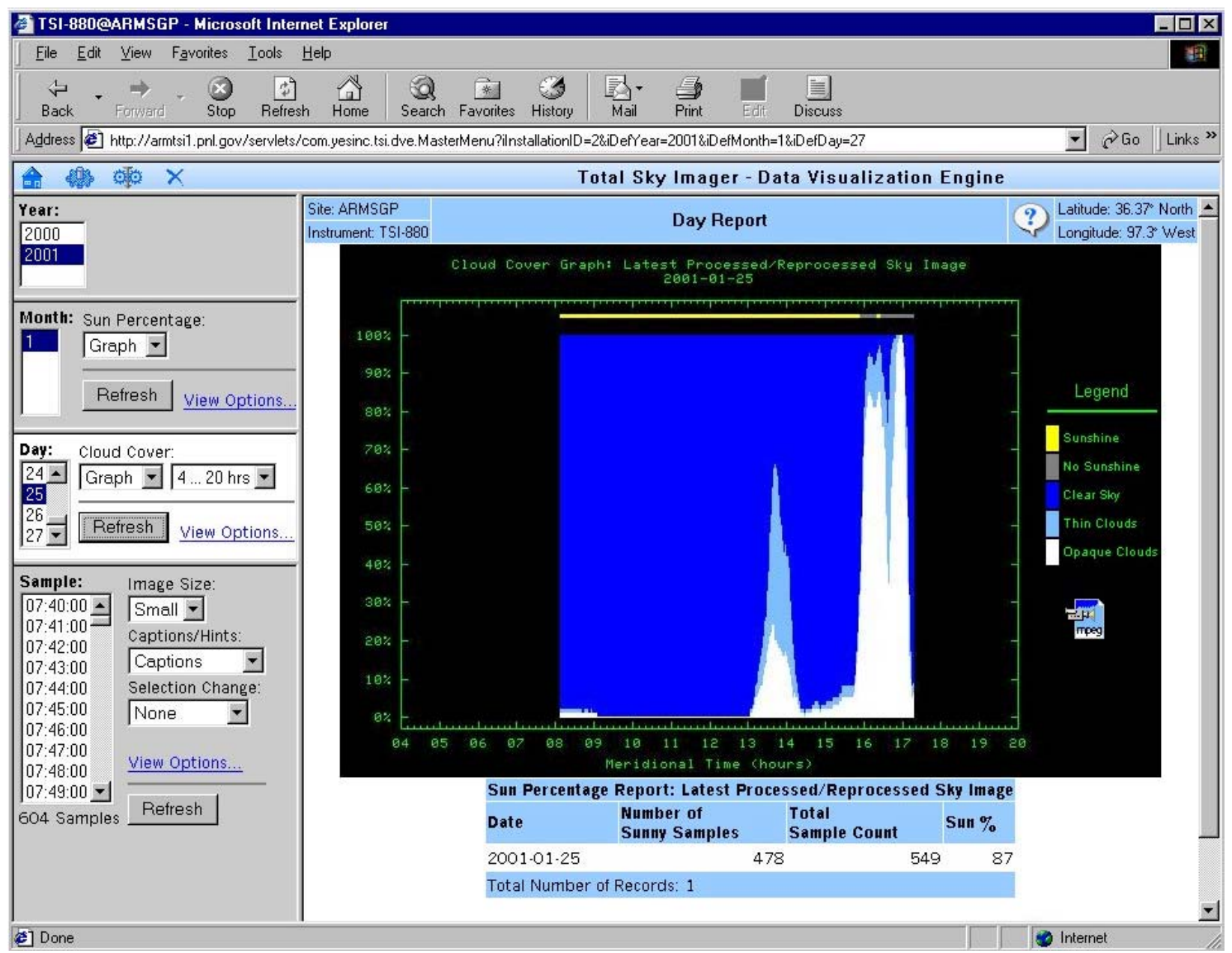

Figure B7. Sample of the TSI 880 Data Visualization Engine Sky Cover Plot Page. 


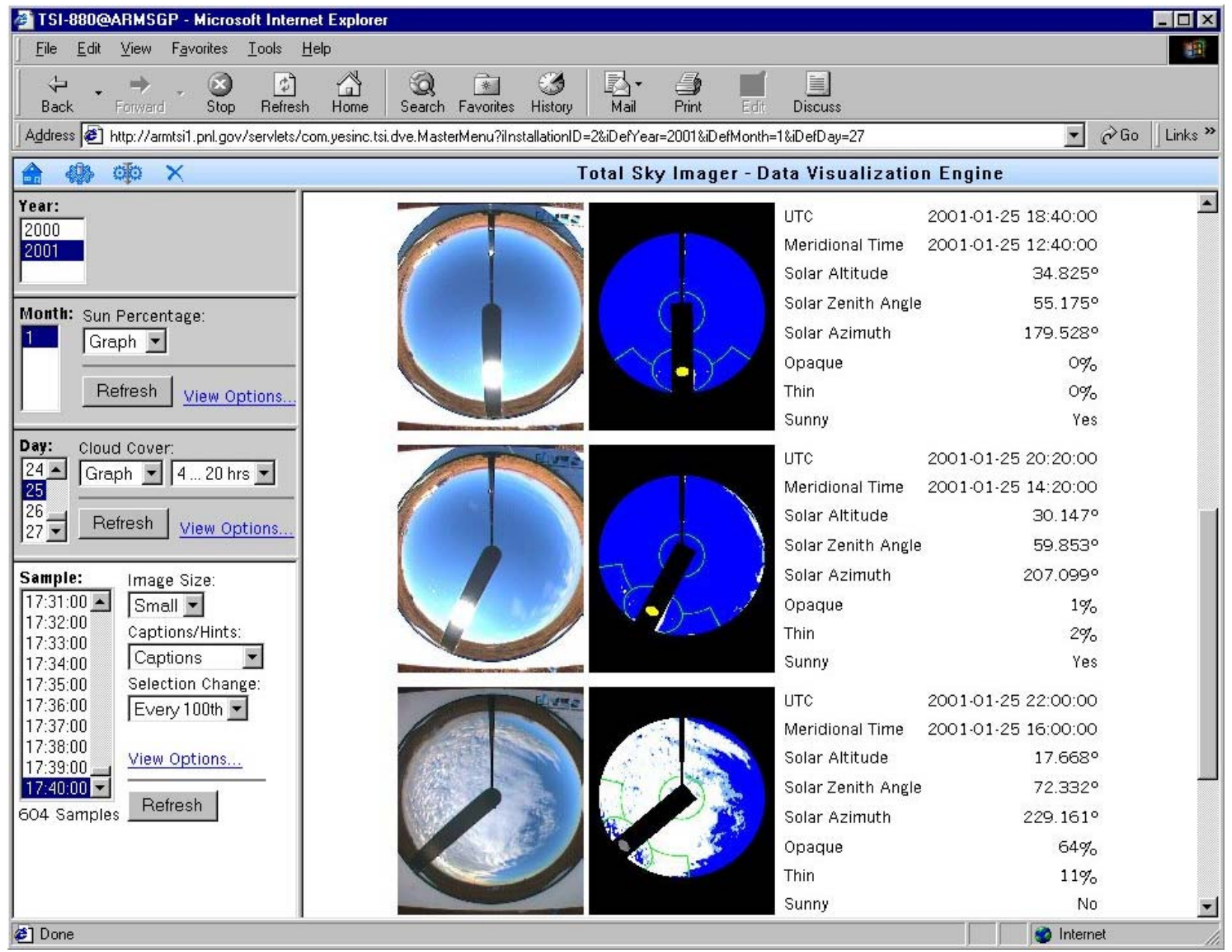

Figure B8. Sample of the TSI 880 Data Visualization Engine Raw And Cloud Decision Image Samples Page. 


\section{Appendix C}

\section{Testing and Evaluation Data Formats}




\section{Appendix C}

\section{Testing and Evaluation Data Formats}

Daily files of the sky images, cloud decision images, and individual image properties files have been bundled as "tar" files, though YES software gives these files a ".dat" extension. These daily tar files use a "YYYYMMDD.dat" naming convention, where

YYYY = 4-digit year

$\mathrm{MM}=$ month

$\mathrm{DD} \quad=$ day of month

Note that after the first month or so of deployment at BTA during the 2000 Cloud IOP, the daily tar files also include other numbers and letters following the date in the stem of the file name, a change in YES format file naming. However, the contents of these longer file names are the same as the date-only stem files.

\section{Sky Images}

Sky images are in JPEG image format, 288 X 352 pixel resolution, 24-bit color. Image file names are in "YYYYMMDDhhmmss.jpg" format, where:

$\begin{array}{ll}\text { YYYY } & \text { 4-digit year } \\ \mathrm{MM} & =\text { month } \\ \mathrm{DD} & =\text { day of month } \\ \mathrm{hh} & =\text { hour of day }(0-23) \\ \mathrm{mm} & =\text { minute of hour } \\ \mathrm{ss} & =\text { seconds }\end{array}$

\section{Cloud Decision Images}

Cloud decision images are in PNG image format, same resolution as the raw JPEG images, with the same file stem naming convention. In these images, black represents ignored (masked) pixels, medium blue represents clear sky, white represents thick (opaque) cloud, and light blue represents thin cloud. The green outlines denote special areas (around the sun, at zenith, and along the horizon centered on solar azimuth) where clear/cloud pixel counts are also noted in the output files. 


\section{Image Properties Files}

Image properties files are in ASCII format, with the same file stem naming convention as the image files. These files contain information on the instrument and processing settings, as well as the processing results, for that image. These files are used for operations and reprocessing, and are not generally of interest for the user.

\section{Fractional Sky Coverage}

1) Original ASCII output files produced by the YES cloud algorithm have the naming format "YYYYMMDD.txt," where YYYYMMDD are the same as noted above. From YES documentation, values included in the files, by column:

- Date/time in human format

- Date in machine format (number of seconds since 1-1-1970)

- opaque cloud percentage

- thin cloud percentage

- sunshine indicator (true/false)

- sunshine "strength" (relative value, from integration of blocking strip luminence)

- solar altitude (degrees)

- solar azimuth (degrees)

- "box" pixel count

- "below horizon" pixel count

- "below processing zenith angle" pixel count

- mask pixel count

- "undetermined" pixel count

- sky pixel count

- and if shipboard,

- latitude (degrees)

- longitude (degrees)

- platform heading (degrees)

- and if sun area,

- Total pixels in sun circle

- Opaque pixels in sun circle

- Thin pixels in sun circle

- and if horizon area, (note that if overlap, sun circle takes precedence)

- Total pixels in horizon area

- Opaque pixels in horizon area

- Thin pixels in horizon area

- and if zenith area, 
- Total pixels in zenith circle

- Opaque pixels in zenith circle

- Thin pixels in zenith circle 